\title{
Characterization of Resistance to Pratylenchus thornei (Nematoda) in Wheat (Triticum aestivum): Attraction, Penetration, Motility, and Reproduction
}

\author{
Katherine Joanne Linsell, Ian Timothy Riley, Kerrie Ann Davies, and Klaus Herbert Oldach
}

First, second, and fourth authors: South Australian Research and Development Institute, GPO Box 397, Adelaide, South Australia, 5001, Australia; first, second, third, and fourth authors: University of Adelaide, School of Agriculture, Food and Wine, South Australia, Australia; and first and fourth authors: Molecular Plant Breeding CRC, Adelaide, South Australia, Australia. Accepted for publication 5 September 2013.

\begin{abstract}
Linsell, K. J., Riley, I. T., Davies, K. A., and Oldach, K. H. 2014. Characterization of resistance to Pratylenchus thornei (nematoda) in wheat (Triticum aestivum): Attraction, penetration, motility, and reproduction. Phytopathology 104:174-187.

Lines from a cross between two wheat (Triticum aestivum) cultivars with contrasting resistance phenotypes to Pratylenchus thornei (Nematoda) were investigated to determine the stage at which resistance occurs. Host resistance was examined at nematode attraction to and penetration of roots and nematode motility, maturation, and reproduction within roots. There was no significant difference in the rate at which $P$. thornei

penetration. No preferential root penetration zone was observed in contrast to other studies. The inhibitory compounds from resistant wheat plants appeared to be constitutively expressed and water soluble because nematode migration was suppressed in roots and root exudates of unchallenged seedlings. The effects of these compounds were reversible and affected $P$. thornei but not $P$. neglectus. Apart from migration, nematode multiplication was greatly inhibited by resistance because only a few juveniles $(10 \%)$ developed past stage three in roots of resistant compared with susceptible plants. Earlier in the life cycle, egg deposition and hatch of $P$. thornei were also significantly reduced in resistant roots and root exudates, suggesting the presence of hatching inhibitors.
\end{abstract} was attracted toward or penetrated resistant or susceptible roots. However, suppression of migration, juvenile maturation, and reproduction in and near resistant roots was evident, suggesting that resistance acts post
Additional keywords: molting, root lesion nematode.
Several Pratylenchus spp. occur in Australia on various hosts, with two dominant in cereals, Pratylenchus thornei and P. neglectus (61). Necrotic lesions form as a result of cell death caused by Pratylenchus nematodes feeding on root cortical cells $(37,79,80)$. Cortical degradation and reduced root branching diminishes the plant's ability to acquire nutrients and water from the soil, stressing the plant and reducing vegetative growth and yield. The value of wheat production lost in Australia due to $P$. thornei is estimated at >\$AUD 50 million each year (39).

The process by which Pratylenchus spp. invade and develop can be separated into four main components: root recognition, penetration, feeding and migration, and reproduction. Initially, the nematodes are attracted to the root and probe the surface for an acceptable penetration site. After a salivation period, the nematode may feed ectoparasitically on root hairs. Using stylet thrusting and body movement, they penetrate the root epidermis (79), enter the root, and migrate intracellularly while feeding endoparasitically on root cortex cells (80). During migration, at each new cell, the nematode punctures the cell wall with stylet thrusts and pushes through into the adjacent cells to continue feeding $(32,79,80)$. While in the roots, adult Pratylenchus nematodes deposit eggs in the cavities created by migration (2). Eggs are also deposited in the soil in close vicinity to the roots $(11,40)$. The juvenile hatches from the egg as a stage-two juvenile (J2) and, at each molt, undergoes further sexual development (52). The

Corresponding author: K. H. Oldach; Email address: klaus.oldach@sa.gov.au

http://dx.doi.org/10.1094/PHYTO-12-12-0345-R

(c) 2014 The American Phytopathological Society full cycle from egg to adult is completed within 45 to 65 days (67) and is greatly influenced by host, temperature, and Pratylenchus sp.

Due to the wide host range of Pratylenchus spp. and the restrictions, costs, and inefficiency of chemical nematicides, the development of resistant cultivars has become increasingly important as a control measure $(1,59)$. Most studies of wheatPratylenchus interactions have focused on the genetic inheritance of resistance $(61,59)$. The identification of new genetic sources of $P$. thornei resistance and the identification of resistance quantitative trait loci (QTL) and associated molecular markers is critical, due to the lack of resistance available in current commercial cultivars. However, knowledge of the biological or biochemical resistance mechanisms is also important because it can provide insights into the nature, timing, and action of resistance genes which could provide new sources of biochemical control and reveal the involvement of different resistance genes, which could be pyramided for a more durable resistance (27). In addition, the understanding of resistance at the biological and biochemical level could be used to improve phenotypic screening methods. Common phenotypic resistance screening techniques are laborious, time consuming, and inherently variable because they involve extracting and counting nematodes from the roots. DNA-based detection methods can achieve higher throughput (42) but are expensive. The identification of a resistance mechanism at a specific stage of invasion or within a particular tissue may enhance screening procedures by reducing these associated costs and time. However, there are gaps in the knowledge of the biochemistry and molecular biology of plant defense responses to migratory endoparasitic nematodes, largely because they cause widespread cellular destruction and lack a fixed feeding site. 
Only a small number of studies have considered the effects of resistance on Pratylenchus biology. Talavera and Vanstone (58) demonstrated that $P$. thornei is able to penetrate resistant cultivars and Farsi (14) observed equal root penetration of $P$. neglectus in both resistant and susceptible wheat lines. Other studies in various plant hosts have shown that, in other Pratylenchus spp., resistance is associated with reduced motility and reproduction $(3,45,48,64)$. It is hypothesized that resistance in wheat to $P$. thornei occurs post root penetration and causes reduced reproduction by either the suppression of migration through the cortex which, in turn, suppresses feeding; the suppression of egg deposition and hatching; or inhibition of nematode maturation (Fig. 1).

In this study, the parents of a cross between two wheat cultivars with contrasting resistance phenotypes to P. thornei, 'Sokoll' (resistant) and 'Krichauff' (susceptible), in addition to six selected lines from the population were investigated. The chosen population lines were selected based on their resistant genotypes as determined by QTL analysis (Linsell, Rahman, Taylor, Davey, Gogel, Wallwork, Forrest, Hayden, Taylor, Oldach, unpublished data). The first aim was to compare the early stages of the invasion process of $P$. thornei, including root attraction, penetration, and nematode motility within the root and in root exudates (REs), in these resistant and susceptible genotypes in order to identify the stages where and how resistance has an effect. The second aim was to determine whether the resistance within these genotypes is due to reduced juvenile development, egg deposition, and egg hatch. The investigation aimed to generate useful screening protocols, gain a better understanding of the biological resistance mechanisms, and provide a foundation for directed biochemical investigations into $P$. thornei-resistant wheat responses.

\section{MATERIALS AND METHODS}

Nematodes. $P$. thornei was obtained from wheat at Nunjikompita, South Australia and cultures were maintained on carrot callus, as described by Moody et al. (38). Cultures were stored at $22^{\circ} \mathrm{C}$ and subcultured every 3 months. To collect the nematodes, carrot callus was placed in funnels in a misting chamber with an intermittent aqueous mist of $10 \mathrm{~s}$ every $10 \mathrm{~min}$ for $96 \mathrm{~h} \mathrm{(56).}$ Nematodes extracted in the mister were counted in $250-\mu \mathrm{l}$ aliquots in three replicates and diluted with water to the required inoculum concentration. Because $P$. thornei is a parthenogenic species, most of the nematodes collected were females. To obtain inoculum of nematodes of a single developmental stage, suspensions were passed repeatedly through sieves. Adults and stagefour juveniles $(\mathrm{J} 4 \mathrm{~s})$ were captured on a $40-\mu \mathrm{m}$ sieve, stage-three juveniles $(\mathrm{J} 3 \mathrm{~s})$ on a $30-\mu \mathrm{m}$ sieve, and $\mathrm{J} 2 \mathrm{~s}$ were retained on a $20-\mu \mathrm{m}$ sieve. Eggs but few nematodes were able to pass through the $20-\mu \mathrm{m}$ sieve. This sieving method provided relatively high purity; nevertheless, the presence of nonrequired stages was recorded.

Plant material. The study investigated a doubled-haploid (DH) population from a cross between the synthetic-wheat derived Sokoll (P. thornei resistant) and the Australian wheat Krichauff (P. thornei susceptible). This population was phenotyped using DNA quantification methods and genetically mapped to identify two highly significant resistant QTL on chromosomes 2B and 6D (Linsell, Rahman, Taylor, Davey, Gogel, Wallwork, Forrest, Hayden, Taylor, Oldach, unpublished data). The parents and six selected individual lines (three susceptible [S1, S2, and S3] and three resistant [R1, R2, and R3]) were analyzed. The six lines were selected based on genotypic and phenotypic data. The three resistant lines had both resistant QTL and the three susceptible lines had neither resistance QTL. The chosen resistant and susceptible selected lines contained the lowest and highest amounts of quantified $P$. thornei DNA, respectively. For all experiments, prior to germination, seed were surface sterilized by submersion in $70 \%$ ethanol for $5 \mathrm{~min}$, then hypochlorite $(15 \%)$ for $20 \mathrm{~min}$ at room temperature (RT) of 21 to $22^{\circ} \mathrm{C}$, followed by four rinses with reverse osmosis ( $\mathrm{RO})$ water.

Nematode inoculation and extrapolation. For experiments on agar, 3-day-old wheat seedlings with a primary leaf and a 20-mm seminal root were placed on $0.5 \%$ water agar in $90-\mathrm{mm}$ petri plates at RT. After $48 \mathrm{~h}$, plates were inoculated with $P$. thornei by pipetting suspensions onto the surface. Nematodes on the agar were counted using a stereomicroscope (M3C; Wild, Heerbrugg,

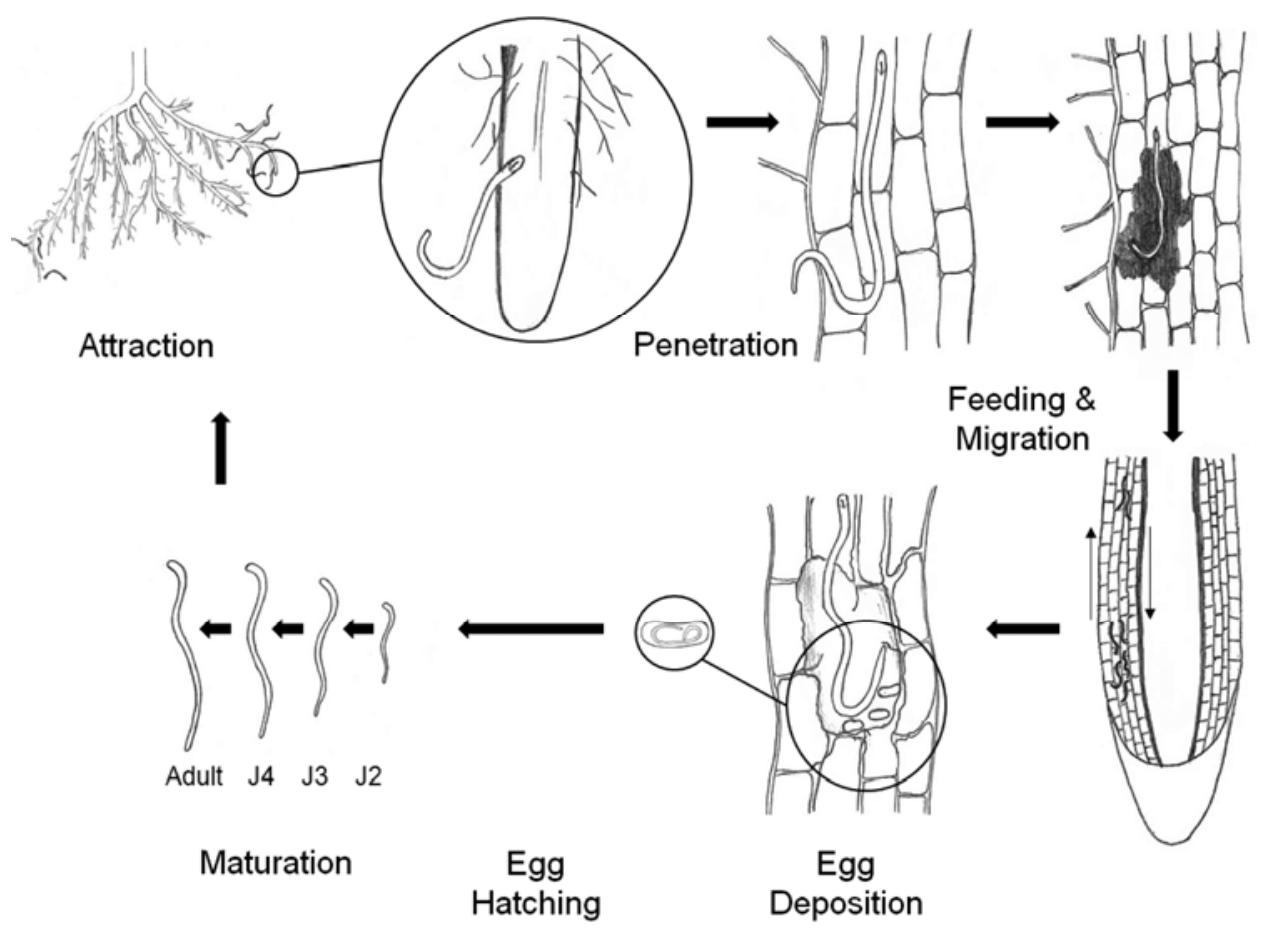

Fig. 1. Life cycle of Pratylenchus thornei associated with Triticum aestivum, with special reference to host resistance characterization stages. 
Switzerland) and a grid template (10-mm block segments). To count nematodes that had entered roots, seedlings were placed in a misting chamber for $96 \mathrm{~h}$ and were extracted and counted. Nematode suspension $(\approx 10 \mathrm{ml})$ was collected and stored at $4^{\circ} \mathrm{C}$ until counted in 500- $\mu$ l aliquots using a stereomicroscope, and the total number in the root system was estimated accordingly. Alternatively, nematodes that could no longer be seen on the agar surface were assumed to have penetrated the roots (initial inoculum number - number of nematodes remaining on agar plate $=$ number of nematodes within roots). This was confirmed in a check experiment at $12 \mathrm{~h}$ after inoculation (hai), where the total number of nematodes extracted from the agar via misting plus the total number that had entered the roots equaled the initial inoculum number.

For experiments in pots, plants were grown in a glasshouse maintained at $20 \pm 3^{\circ} \mathrm{C}$. One $\mathrm{DH}$ seed was sown in steampasteurized sand in each seedling tube $(55$ by $120 \mathrm{~mm}$ ) in a fiveby-five randomized block arrangement. Seven days after planting, each seedling was inoculated with $P$. thornei by pipetting two $500-\mu \mathrm{l}$ aliquots of nematode suspension into two 50-mm-deep holes on either side of the seedling. Inoculum densities were specific for each experiment and are described later. At 2 days after inoculation (dai), slow-release fertilizer (Osmocote; Scotts Miracle-Gro) was added ( $4 \mathrm{~g} / \mathrm{kg}$ of sand) to the tubes. Plants were hand watered every 2 days to soil water holding capacity. Nematodes were extracted by placing the washed root systems into a misting chamber, as described above.

Attraction. Movement of $P$. thornei in response to the presence of roots of susceptible and resistant genotypes was investigated in sand and on agar systems. For all experiments on agar, it was assumed that nematodes that could no longer be seen had penetrated roots and were expressed as a percentage of the initial inoculation number.

Attraction on agar. Five replicates of 5-day-old seedlings of both parents were placed on $0.5 \%$ water agar. Plates were divided into $10-\mathrm{mm}$ segments, with roots occupying $30 \mathrm{~mm}$ at one end. In the first $20 \mathrm{~mm}$ at the opposite end to the roots, inoculum of 150 mixed-stage $P$. thornei was added (Fig. 2A). A no-root control plate was included with the same plate design but excluding active seedling roots. The number of $P$. thorne $i$ on each plate was counted. At 6, 9, 12, 27, 30, 33, 36, and 48 hai, the number of nematodes in each segment was counted and their location on the plate grid recorded, reflecting the distance nematodes had moved toward the roots over time. The assay was repeated twice.

Attraction on agar across REs. Six seedlings for both Sokoll and Krichauff were grown aseptically in 14-ml glass vials on nylon mesh in $8 \mathrm{ml}$ of sterile RO water. RE was collected after roots had been submerged in water for 7 days. Ten independent replications were collected and the experiment was repeated twice. A known susceptible seedling ('Machete') was placed at one end of the $0.5 \%$ water agar plate to stimulate nematode movement. RE was pipetted onto the plate in front of the root system so that, in order to reach the roots, the nematodes had to move across the RE solution. The same gridded plate described above (Attraction on agar) was used but $1 \mathrm{ml}$ of RE was placed in a $20-\mathrm{mm}$ segment $30 \mathrm{~mm}$ from the plate edge (Fig. 2B). Plates were inoculated with 500 mixed-stage $P$. thornei $30 \mathrm{~mm}$ from the $\mathrm{RE}$ and $30 \mathrm{~mm}$ from the plate edge so that nematodes could either move toward or away from the exudates. After $48 \mathrm{~h}$, the number and location of individual nematodes on the plates was assessed. The numbers of nematodes that remained at the point of inoculation, which moved away from the root (repelled), and moved toward the root (attracted) were expressed as a percentage of the initial inoculation number.

Attraction with both genotypes present. Sixteen different resistant versus susceptible combinations made from the parents and the six DH lines were assessed either in sand or on agar. These same combinations were twice analyzed independently both in sand and on agar. For experiments in sand, one resistant and one susceptible seedling was sown into the same pot (55 by $120 \mathrm{~mm}$ ) and inoculated with two 500- $\mu \mathrm{l}$ aliquots (500 nematodes/pot) into two 50-mm-deep holes between the two seedlings. Roots were harvested after 14 days, freed from sand, and placed on the mister for nematode extraction. For experiments on agar, resistant and susceptible 5-day-old seedlings were placed at either end of a $90-\mathrm{mm}$ plate with $0.5 \%$ water agar. The plate was inoculated with 225 mixed-stage nematodes in a 30-by-10-mm rectangle near its center (Fig. 2C). After 9 days, roots were placed on a mister for nematode extraction and collection. Attraction was assessed by measuring the percent penetration of the resistant and susceptible root in each combination. Then, the number of combinations that had more penetration in the resistant or susceptible roots was calculated and presented as a percentage of the total number of combinations analyzed.

Penetration. Rates of penetration in sand and on agar. The rate of $P$. thornei penetration was assessed both in sand and on agar systems. For experiments in pots, seedlings were grown in sand under glasshouse conditions $\left(20 \pm 3^{\circ} \mathrm{C}\right)$ and were inoculated with 1,500 mixed-stage $P$. thornei nematodes/plant. Roots were harvested at 5, 7, 10, and 16 dai, washed, and placed on the mister. The parents and two lines from each of the resistant and susceptible lines were assessed with five replicates. For agar analysis, 3-day-old seedlings that had been germinated on moistened sterile filter paper were placed at one end of a plate ( $0.5 \%$ water agar) and inoculated with 150 mixed-stage nematodes/plant at the opposite end. Three independent analyzes were conducted using three replicates of the parents and three lines of both resistant and susceptible genotypes. Nematodes visible on the plate were
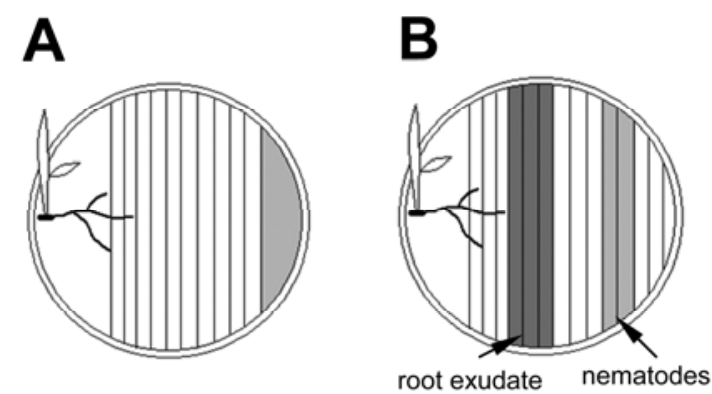
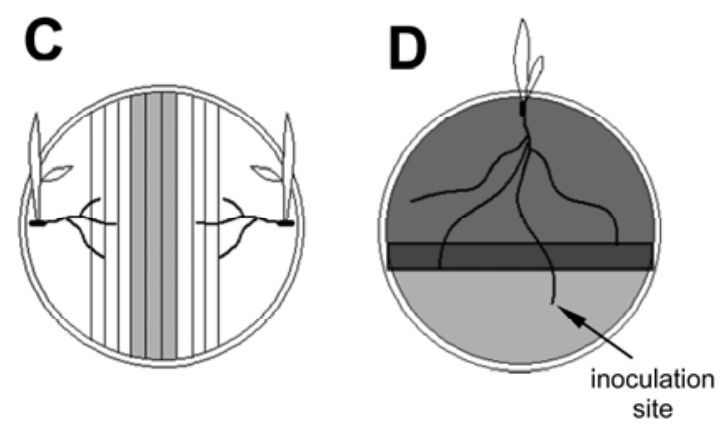

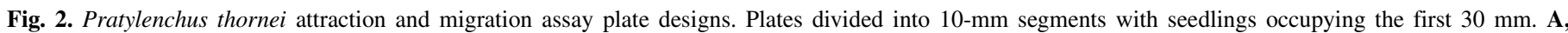

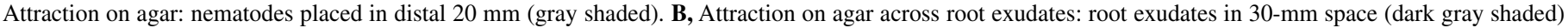

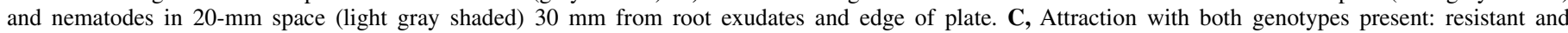

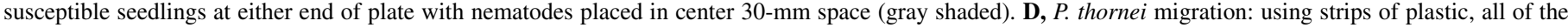

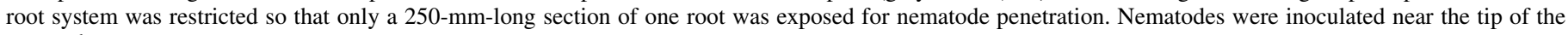
exposed root. 
counted at $6,9,12,30$, and 36 hai. The number of nematodes that had penetrated roots was calculated (initial inoculum number number visible on plate at time point) and means were expressed as a percentage of the initial inoculation number.

Penetration of adults versus juveniles on agar. Differences in rates of penetration of adults and juveniles was assessed in sand and agar systems. For experiments in sand, seedlings were inoculated with 500 adult nematodes/plant or $500 \mathrm{~J} 2$ nematodes/plant. Roots were harvested at 3, 4, 6, and 8 dai. Two resistant and susceptible lines and both parents were assessed with five replications. For experiments on agar, seedlings on $0.5 \%$ water agar were inoculated with 1,000 nematodes/plant and the numbers of visible nematodes were counted at $6,12,24$, and 30 hai. The number of $P$. thornei that penetrated roots was calculated and means at each time point were expressed as a percentage of the initial inoculation number.

Impact of crushed root suspensions and REs on motility. The effect of resistance on $P$. thornei motility was investigated with in vitro assays in REs and crushed root (CR) suspensions. A nematode was considered nonmotile if no movement was observed within $5 \mathrm{~s}$. The concentration of RE and CR suspensions were standardized to minimize variation between roots, because larger root systems are expected to produce more REs and contain more root compounds when crushed than smaller roots. Thus, all Res or suspension samples were adjusted to a set optimum concentration of $4 \mathrm{mg} / \mathrm{ml}$ of fresh weight root tissue in water, as determined by testing motility suppression in an RE dilution series.

REs. Seedlings were grown aseptically in petri plates on sterile filter paper for 10 days at RT. Three replicates of each of the six $\mathrm{DH}$ lines and the parents were analyzed. $\mathrm{RO}$ water $(1 \mathrm{ml})$ was used to wash the roots, and filter paper and the liquid was collected. Fresh roots were weighed and the collected RE adjusted to a concentration of $4 \mathrm{mg} / \mathrm{ml}$ of fresh weight root tissue by addition of RO water. A 100- $\mu$ l aliquot of RE was transferred to individual wells $(350 \mu \mathrm{l})$ on a 96-well enzyme-linked immunosorbent assay (ELISA) plate and inoculated with $\approx 80$ mixed-stage nematodes. Three replicates of each sample were assessed. Numbers of motile and nonmotile nematodes were counted at 1,2, and 3 dai at RT. These assays were performed on both $P$. thornei and P. neglectus.

To determine whether the effect of resistant RE on $P$. thornei motility could be reversed, nematodes were recovered from the resistant Sokoll (10 samples) RE after 1 day of exposure into water, and motility was reassessed after 2 days.

CR suspensions. One seed was sown in each seedling tube (55 by $120 \mathrm{~mm}$ ) in steam-pasteurized sterilized sand. Three replicates of each of the four DH lines (two resistant and two susceptible) and the parents were analyzed. Plants were grown for 1 week in a glasshouse maintained at $20 \pm 3^{\circ} \mathrm{C}$ and were hand watered every 2 days with $100 \mathrm{ml}$. Plants were then washed and fresh root weights were recorded. Roots were crushed in liquid nitrogen and immediately suspended in RO water. The CR suspensions were centrifuged and supernatant removed and adjusted to a concentration of $4 \mathrm{mg} / \mathrm{ml}$ of fresh weight root tissue by addition of RO water. Experiments were conducted either in $6 \mathrm{ml}$ of CR suspension in sample containers ( 40 by $45 \mathrm{~mm}$ ) or $100 \mu \mathrm{l}$ of CR suspension in wells on an ELISA plate. Each replicate was inoculated with $\approx 80$ mixed-stage nematodes. Numbers of motile and nonmotile nematodes were counted using a stereomicroscope (M3C; Wild) at 3 and 7 dai at RT.

Migration. The effect of resistance on $P$. thornei migration within the root cortex was investigated with a root exclusion assay on agar. Seedlings were germinated on sterile filter paper and then placed on $0.5 \%$ water agar for 3 to 4 days at RT. Three replicates of each of the six DH lines and the parent sample were analyzed. Using strips of plastic, all of the root system was blocked off except a $250-\mathrm{mm}$ section of one root. Water agar (1\%) was then used to cover the rest of the roots and the plastic strips acted as a wall so that only the $250-\mathrm{mm}$ root portion was exposed for nematode penetration (Fig. 2D). In all, $\approx 100$ mixed-stage nematodes were inoculated near the exposed root. At 5 dai, the seedling was removed from the plate and the roots were stained in acid fuchsin (7). Stained roots were placed in petri plates and, using a microscope, the inoculation point and the distance nematodes had migrated from that point on the exposed root was marked on the plate and measured.

Reproduction and maturation. $P$. thornei development within resistant and susceptible roots was assessed in sand over 45 days (one generation). Five replicates of the parents and six DH lines (three susceptible and three resistant) were grown in glasshouse conditions, as described above. Each plant was inoculated with $500 \mathrm{~J} 2 \mathrm{~s}$. Roots were harvested at 5, 10, 15, 24, 30, 36, and 45 dai and nematodes were extracted by misting. These times were chosen based on the timeline observed by Larson (33). The number of $\mathrm{J} 2, \mathrm{~J} 3$, and $\mathrm{J} 4$ or adult $P$. thornei present at each of these times was counted and means at each time point were calculated. Developmental stages were assessed by size after separation through sieving and counted with a dissecting microscope. The $\mathrm{J} 4 \mathrm{~s}$ and adults were counted as one group. The maturation data set had a non-normal distribution and was transformed using natural logs.

Egg deposition. The effect of resistance on $P$. thornei egg deposition was investigated by observing mature females in the presence of roots on agar. Five replicates of the parents and three DH resistant and susceptible lines were assessed. Seven-day-old seedlings on water agar $(0.5 \%)$ were inoculated with $1,000 \mathrm{~J} 4$ and adult $P$. thornei nematodes adjacent to the roots. No eggs were present. After 10 days at RT, the numbers of eggs on the agar were counted under a stereomicroscope. The roots were then stained in acid fuchsin, and eggs deposited by adults that had entered the roots were counted.

Hatching. The rate of $P$. thornei juvenile hatching was assessed by exposing mature eggs to roots grown on agar and to RE with the same set of lines as for egg deposition in five replicates.

For analysis on agar, seed were germinated on moist filter paper and 6-day-old seedlings were transferred to water agar $(0.5 \%)$ media plates and inoculated with $80 \mathrm{P}$. thornei eggs directly at the roots. At RT 8 dai, the hatched juveniles on the agar were counted. To count juveniles that had migrated into the roots, juveniles were extracted by misting (misting chamber) or roots were stained with acid fuchsin.

For analysis in RE, seedlings were grown aseptically in petri plates on sterile filter paper for 12 days at RT. REs were taken from young plants because $P$. thornei hatching was shown to be greater in RE from younger plants (47). RO water $(1 \mathrm{ml})$ was used to wash the roots and filter paper and the liquid was then collected. Fresh roots were weighed and collected exudates were adjusted to $4 \mathrm{mg} / \mathrm{ml}$ of root tissue. The RE (100 $\mu \mathrm{l})$ was transferred to individual wells on a 96 -well ELISA plate. In all, $\approx 80$ eggs were added to each well $(350 \mu \mathrm{l})$ and the numbers of hatched J2s were counted at 3, 7, and 10 dai. Hatching assays were also performed in dilutions of 4,10 , and $100 \mathrm{mg} / \mathrm{ml}$ of root tissue to determine the presence of hatching inhibitors (HIs). Five replicates of the parents and three DH resistant and susceptible lines were assessed twice. Because Pratylenchus hatching is influenced by temperature, experiments were performed at the optimum temperature of 21 to $22^{\circ} \mathrm{C}(10)$.

Statistical analysis. Replicate means were calculated and were pooled for each sample from each independent assay to give one data set for each experiment. Normally distributed data sets were analyzed by analysis of variance (ANOVA) with Fisher's least significant difference post-hoc test to compare means, where $P<$ 0.05 was considered statistically significant using the statistical program Genstat (VSN International). Correlation coefficients were calculated and used to interpret trends. Prior to ANOVA 
analysis, data sets were checked for normality and homogeneity of variances, and were log-transformed where necessary to correct deviations from these assumptions.

\section{RESULTS}

Attraction. Attraction on agar. The rate at which $P$. thornei moved across the agar toward the roots over $36 \mathrm{~h}$ was not significantly $(P=0.05)$ different between genotypes. At each of the seven intervals assessed $(6,9,12,27,30,33$, and 36 hai), there were equal numbers of $P$. thornei in each grid (data not shown). After 36 hai, the total number that had reached and entered the root was not significantly $(P=0.05)$ different.

Attraction on agar across RE. This assay measured nematode movement in response to resistant and susceptible RE. To reach the root system of the susceptible Machete (which acted to stimulate nematode movement), nematodes had to move through RE. Eight Sokoll, seven Krichauff, and six no-root control replications were analyzed. At 48 hai, there was no statistically significant difference between nematode movement away or toward the RE within or between genotypes (Table 1). Generally, $23 \%$ (115 nematodes) were attracted to the roots and $\approx 15 \%$ (78 nematodes) were repelled. The majority, with $65 \%$ or 325 nematodes, remained at the point of inoculation. In the no-root control where there was no RE barrier, a similar number moved away and toward the roots.

TABLE 1. Percent Pratylenchus thornei movement on agar in response to root exudates from the resistant (Sokoll) and susceptible (Krichauff) Triticum aestivum genotypes $48 \mathrm{~h}$ after inoculation

\begin{tabular}{lcccc}
\hline Genotype & $n$ & Attracted $(\%)$ & Repelled $(\%)$ & Stationary $(\%)$ \\
\hline Sokoll & 8 & 22.6 & 16.6 & 70.7 \\
Krichauff & 7 & 23.3 & 14.3 & 60.8 \\
No-root control & 6 & 15.1 & 14.2 & 62.4 \\
Standard error & $\ldots$ & 9.2 & 7.7 & 7.6 \\
\hline
\end{tabular}

TABLE 2. Attraction of Pratylenchus thornei (measured as penetration) to 16 different resistant and susceptible Triticum aestivum combinations assessed in sand (14 days after inoculation) and on agar (9 days after inoculation), where roots of both genotypes were available for nematodes to penetrate

\begin{tabular}{lccl}
\hline Penetration $(\%)$ & Resistant & Susceptible & $n$ \\
\hline Sand & 52.2 & 47.8 & 13 \\
Standard error & 9.1 & 5.2 & $\ldots$ \\
Agar & 16.7 & 81.3 & 14 \\
Standard error & 4.4 & 4.4 & $\ldots$ \\
\hline
\end{tabular}

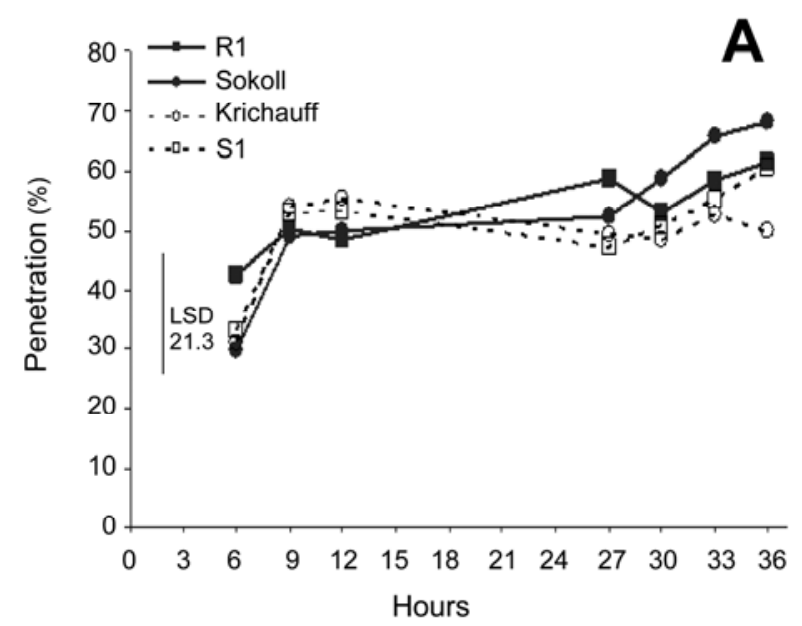

Attraction with both genotypes present. Across two repeated experiments, 16 different resistant and susceptible combinations, including the parents and DH lines (S1, S2, S3, and Krichauff versus R1, R2, R3, and Sokoll) were assessed in sand and agar for their ability to attract $P$. thornei. Some replications were excluded due to poor plant growth, leaving data from 13 combinations in sand and 14 on agar to be analyzed. When both resistant and susceptible roots were available for penetration on agar, significantly $(P=0.05)$ more $P$. thornei were attracted to the susceptible roots $(80 \%) 9$ dai; however, in sand, attraction to both genotypes 14 dai $(50 \%)$ was the same (Table 2). On average, in 11 of the 14 different resistant and susceptible root combinations tested, more $P$. thorne $i$ were attracted toward and penetrated the susceptible roots and, in three combinations, more $P$. thornei entered the resistant roots.

Penetration. Rates of penetration in sand and on agar. The penetration on agar was much quicker and was measured in hours whereas days where necessary for the sand system. There was no relationship between the rate of $P$. thornei penetration and genotype on agar or in sand when measured 36 hai and 16 dai, respectively. However, there was a significant increase in penetration over time in both genotypes (Fig. 3). Significantly $(P=0.05)$ higher penetration occurred in the agar system than in sand, with $>50 \%$ penetration occurring after only 9 to 10 hai, whereas penetration in the sand system never reached $50 \%$.

Penetration of adults versus juveniles on agar. There was no difference in the rates of $\mathrm{J} 2$ or adult penetration between genotypes. However, comparison of adult and juvenile penetration showed a significant relationship between developmental stage and penetration rate, with a correlation coefficient of 0.4 in both media. On agar, the rate of juvenile penetration was higher than adults but, in sand, it was reversed (Fig. 4). At 30 hai on agar, J2 penetration was almost $40 \%$ more than adult penetration. In comparison, at 8 dai in sand, adult penetration was $30 \%$ higher than $\mathrm{J} 2$, with only $7 \%$ total $\mathrm{J} 2$ penetration.

Root penetration zones. P. thornei nematodes were observed probing the root surface in various root zones but were most frequently detected at the junction of lateral branches and seminal main root axes (Fig. 5A to D). In addition, large groups were often observed both in the zone of elongation (Fig. 5E) and in seminal roots near the hypocotyl. P. thornei nematodes were seen within the dense root hair regions of both seminal main axes (Fig. $5 \mathrm{~F}$ ) and lateral branches but penetration in this zone was not observed. Once the nematodes had penetrated the root, large groups of $P$. thornei at various developmental stages, including eggs, were often seen aggregated in different regions of the root (Fig. 5G).

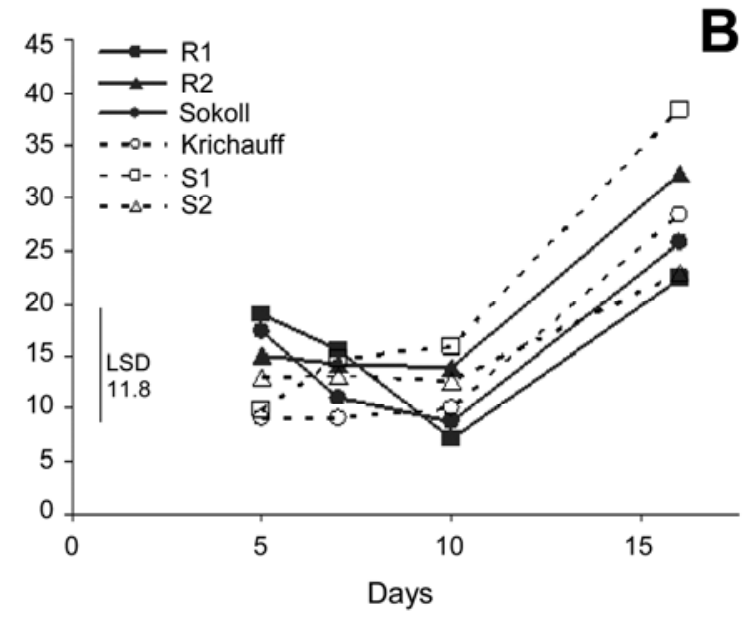

Fig. 3. Triticum aestivum root penetration of Pratylenchus thornei on agar and in sand over A, $36 \mathrm{~h}$ and $\mathbf{B}, 16$ days, respectively, in resistant (Sokoll, R1, and R2) and susceptible (Krichauff, S1, and S2) genotypes. LSD = least significant difference. 
Motility and migration. The motility suppression of RE at 2, 4 , and $100 \mathrm{mg} / \mathrm{ml}$ was assessed and the $4 \mathrm{mg} / \mathrm{ml}$ concentration was found to produce the best differentiation between the resistant and susceptible genotypes (Table 3 ) and, thus, was used in all motility experiments.

There was greater $P$. thornei motility suppression in both resistant $\mathrm{RE}$ and $\mathrm{CR}$ suspensions after exposure for 3 days than in suspensions of susceptible genotypes (Fig. 6A and B). A small amount of motility suppression occurred in Krichauff and the susceptible lines (30\%) but was equal to the no-root control. The resistant Sokoll RE and CR suspensions caused significant loss of motility at 65 and $75 \%$, respectively. In order to determine the specificity of the observed motility suppression, assays were also conducted on the closely related species $P$. neglectus. There was no effect on $P$. neglectus motility when exposed to RE for 3 days because motility suppression was equivalent to the no-root control (25\%) (Fig. 7). The effect of resistant root compounds appeared partially reversible in Sokoll RE because recovery in water, after 2 days of exposure, resulted in almost $50 \%$ of $P$. thornei that had been immotile regaining motility. In the resistant Sokoll RE after 1 day of exposure, motility suppression was $94 \%$. The ability of $P$. thornei to migrate within resistant roots was also suppressed (Fig. 8). Migration within the root was significantly $(P=0.05)$ lower in resistant root genotypes (Sokoll, R1, R2, and R3) than in susceptible (Krichauff, S1, S2, and S3). On average, the migration of $P$. thornei from a set point of penetration was $10 \mathrm{~mm}$ in Sokoll compared with $68 \mathrm{~mm}$ in Krichauff.

Reproduction and maturation. Nematode development in the susceptible lines showed the molt of $\mathrm{J} 2$ to $\mathrm{J} 3$ taking 7 to 10 days and subsequent molts to $\mathrm{J} 4$ and adult occurring within 18 to 28 days. Egg deposition occurred within 2 days after observing mature females, and $\mathrm{J} 2$ hatched 7 to 10 days after.

When comparing the developmental time frames between resistant and susceptible genotypes at each developmental stage, there were significantly $(P=0.05)$ higher numbers of $P$. thornei in the susceptible Krichauff parent than the resistant Sokoll (Fig. 9). Nematode numbers were $\log$ transformed $[\ln (x+1)]$ and are hereafter referred to as $\log$ units followed by $\left(e^{\mathrm{x}-1}\right)$. There was no second-generation hatch of $\mathrm{J} 2$ at 36 dai (Fig. 9A) in Sokoll or any of the resistant lines but, in Krichauff, a mean of 1.24 (13.3) juveniles and, on average, 3.4 (30) juveniles resulted in the susceptible lines. The inoculated $\mathrm{J} 2$ s molted to $\mathrm{J} 3 \approx 10$ dai but no significant difference in numbers occurred between genotypes (Fig. 9B). However, at 45 dai, the numbers of $\mathrm{J} 3 \mathrm{~s}$ in the second generation was significantly $(P=0.05)$ higher in Krichauff at 4.08 (52.3) and the susceptible lines C01 and C35 than in Sokoll at 0.9
(5.3) and the three resistant lines. The J3s molted to J4 and adult at $\approx 15$ to 26 dai The number of $\mathrm{J} 4 \mathrm{~s}$ and adults measured at 24 dai (Fig. 9C) was 10 times higher in Krichauff at 2.58 (44.7) than in Sokoll at 0.64 (4.7). Thus, juvenile development was suppressed as early as the molt from $\mathrm{J} 2$ to $\mathrm{J} 3$.

Egg deposition and egg hatch. The number of eggs deposited by adults placed directly adjacent to roots of seedlings on agar after 10 days was statistically higher in the presence of susceptible genotypes than resistant. The mean number of eggs deposited in or near Krichauff was 53 compared with 18 in Sokoll, giving an egg deposition rate of 4.7 and 1.5 eggs/day, respectively (Fig. 10A and B). Significantly $(P=0.05)$ more eggs were deposited within susceptible roots $(83 \%)$ than outside $(17 \%)$ whereas $50 \%$ of eggs were laid both within and outside the resistant roots. Only 4 eggs were laid in the absence of roots.

Resistant genotypes also stimulated significantly $(P=0.05)$ less hatching than susceptible genotypes. After 10 days, the mean number of juveniles hatched from eggs placed adjacent to resistant Sokoll and susceptible Krichauff roots was 4 and 48, respectively, giving respective hatching rates of 0.5 and 5 juveniles/ day. There was no statistically significant difference in the position of juveniles (within or outside roots) after 10 days between genotypes (Fig. 11A). There were high hatching rates $(45 \%)$ in the no-root control, similar to that of Krichauff. Exposure of eggs to RE suppressed hatching in the resistant genotypes, with $17 \%$ hatch in Sokoll and $25 \%$ in Krichauff after 3 days (Fig. 11B). In the no-root water control, only $8 \%$ hatch occurred. The dilution of RE corresponded to an increase in hatching in both genotypes. There was statistically significant greater egg hatch at a concentration of $4 \mathrm{mg} / \mathrm{ml}$ compared with $100 \mathrm{mg} / \mathrm{ml}$ in all genotypes (Fig. 12).

\section{DISCUSSION}

Root attraction and penetration. Recognition of and attraction to host roots are essential steps that precede nematode root invasion. Host recognition is thought to involve signals from the root that can stimulate egg hatch or attraction toward the roots $(44,46)$. It is generally accepted that nematodes locate roots through chemotactic factors diffused from the host (78). It has been suggested that signal elicitors from the host are water soluble so that they can move through soil toward the nematode (45). REs are regarded as the most probable stimuli in attracting Pratylenchus spp. to the root region $(5,70,71)$. However, gradients in temperature, electrochemical potential, $\mathrm{pH}$, and carbon dioxide also play a role (46). Specific nematode repellents or attractants
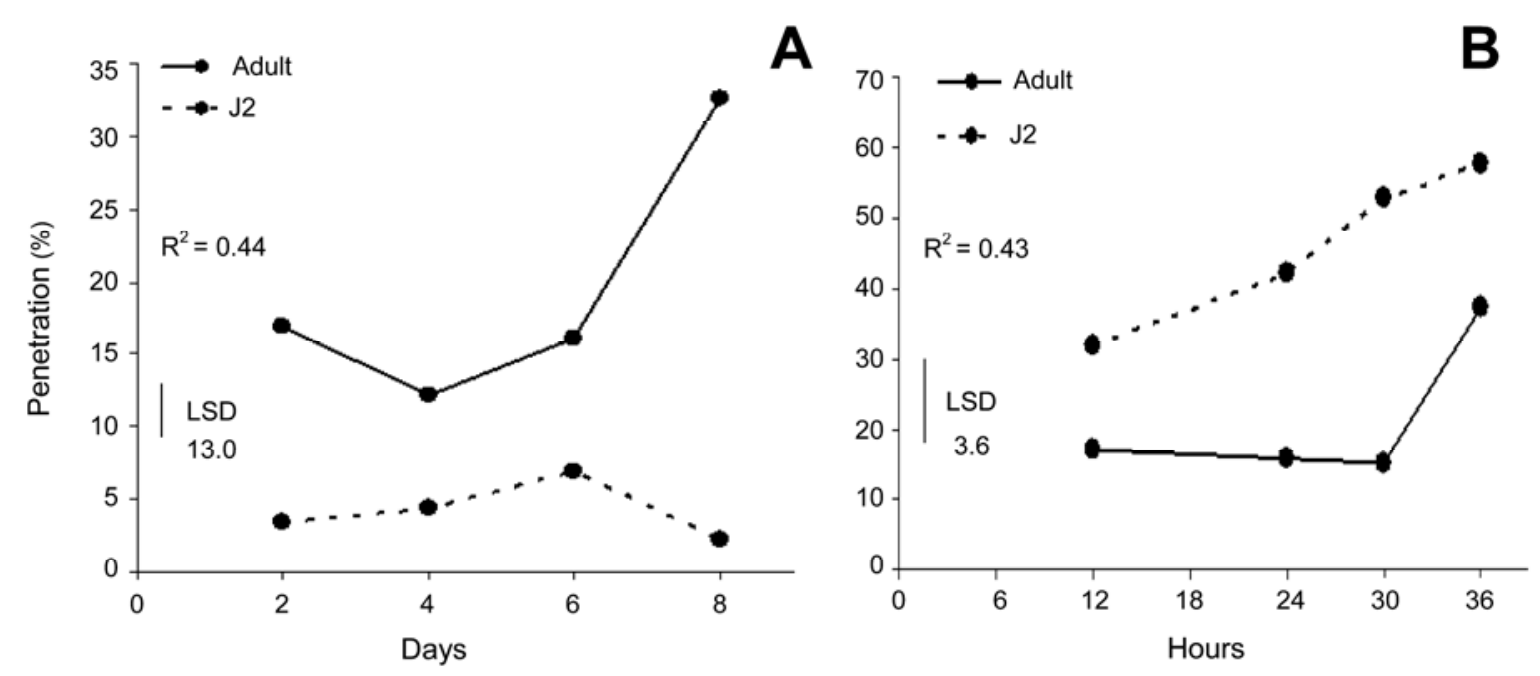

Fig. 4. Triticum aestivum root penetration of second-stage juvenile (J2) or adult developmental stages of Pratylenchus thornei A, in sand and B, on agar over 8 days and $36 \mathrm{~h}$, respectively. $\mathrm{LSD}=$ least significant difference. 
have not been identified but may include amino acids, sugars, phenolics, and other secondary metabolites (18).

REs contain a mix of positive and negative effectors which can attract, repel, or be neutral in terms of nematode behavior $(49,76,77)$. Thus, the effects of REs depend on the combination of signals at a specific time $(19,68,73)$. In this study, there was no difference in the rate (both number and speed) at which $P$. thornei was attracted toward $(23 \%)$ or away $(15 \%)$ from resistant or susceptible roots when only one genotype was present. In addition, in the sand media, when both resistant and susceptible roots were available for penetration, no preference was observed for $P$. thornei attraction after 9 days. This suggests that wheat REs have a neutral effect on $P$. thornei attraction. Other studies, which utilized similar agar and sand biological systems, have also observed that resistance does not interfere with nematode root attraction. For example, Meloidogyne incognita migration to resistant and susceptible cotton roots in sand and $M$. naasi juveniles attraction to resistant and susceptible wheat and barley roots on agar were equal in both genotypes $(4,36)$.

Contrasting observations have been made, however, and preferential attraction has been observed to susceptible roots when both resistant and susceptible genotypes are present in other nematode-plant interactions when analyzed in soil. Bias toward the susceptible roots (72\%) was observed in M. hapla when juveniles hatched between the resistant and susceptible lucerne (alfalfa) roots grown in soil. However, when only one root was present, there was equal attraction and penetration (20). Collectively, these results show that, in some interactions, plants can influence nematode movement toward roots in positive and negative ways. Preferential attraction was also observed in this study but only when attraction was investigated on agar. When both resistant and susceptible roots were present, significantly higher $P$. thornei attraction $(>81 \%)$ occurred toward the susceptible roots 24 hai The bias toward susceptible roots suggests that, when given a choice of both resistant and susceptible roots, either resistant root secretions deter $P$. thornei because they secrete repellent or toxic compounds early during prepenetration, as suggested by Berge (6), or the susceptible roots secrete compounds making them more attractive. The susceptible roots might not produce the repellent compound or produce it in lower concentrations than the resistant roots. When penetration on agar was measured again at 36 hai, there was no difference between resistant and susceptible roots, implying that $P$. thornei may be able to tolerate the effects of resistant root chemicals by activating a protective mechanism. Similar to the observations here, in a resistant potato cultivar, few M. fallax juveniles penetrated the roots 24 hai but, at 60 hai, penetration numbers were comparable with the susceptible cultivar (31). This suggests that the active compounds in resistant root secretions may only influence nematode movement for a limited period, possibly by a potential adaptation of the nematode.

The attraction bias to susceptible roots observed only in agar systems in the presence of both resistant and susceptible genotypes may be due to general differences between the experimental systems in sand (pots) and agar. Due to a greater concentration of active compounds in the small agar volume $(35 \mathrm{ml})$ compared with the larger pot volume $(6,600 \mathrm{ml})$, and with the agar system only being possible with young seedlings, a clear difference in the plants' age exists between agar and sand systems.

Because plant-feeding nematodes possess stylets and generally secrete cell-wall-degrading enzymes (51), they commonly successfully penetrate the host regardless of whether further development occurs. Thus, most nematodes seem capable of overcoming the physical protection barriers of plants. Resistance to root knot, cereal cyst, and root lesion nematodes investigated in several crop species has never been associated with inability to penetrate roots. Juvenile $M$. incognita in cotton and lucerne and several Heterodera spp. in wheat, soybean, and potato were able to successfully penetrate and migrate to the feeding site in both susceptible and resistant cultivars $(12,30,74)$. In addition, similar penetration rates were observed in susceptible and resistant bean cultivars when inoculated with $P$. scribneri 24 hai Thus, it was hypothesized that penetration suppression plays no role in resistance of wheat to Pratylenchus spp. Accordingly, in this study, juvenile $P$. thornei penetration rates did not significantly differ for resistant and susceptible roots both on agar and in sand, even after 16 days. This is supported by Talavera and Vanstone (58), who observed $P$. thornei penetrating resistant wheat cultivars, and Farsi (14), who found no difference in penetration rates of $P$. neglectus between resistant and susceptible wheat cultivars. Neither attraction to roots nor penetration in sand was negatively affected by resistant genotypes; therefore, resistance to $P$. thorne $i$ in the resistant lines investigated here seems to act post penetration.

Penetration of developmental stages. All Pratylenchus developmental stages are able to penetrate and invade roots. However, the rate of penetration by adults in this investigation was significantly higher than that of $\mathrm{J} 2$ penetration in sand. Comparable observations were made in Lucerne, where $P$. penetrans adult females were more invasive than juveniles because they penetrated roots earlier, faster, and over a wider range of soil temperatures (62). The greater ability of adults to penetrate the strong cell walls is attributed to the size of the glands in the posterior subventral lobe, a more developed stylet, and a larger-sized body (one-third larger) compared with a J2 $(24,75)$.

In contrast to the observations in sand, penetration on agar was significantly higher for $\mathrm{J} 2 \mathrm{~s}$ than adults, which can be explained by the differences in the three-dimensional structures of the substrates sand and agar. The higher penetration rates on agar may be due to their increased ability to move across this medium. Nematode movement in soil has been shown to be directly dependent on the size of soil pores (69). Zunke (80) observed that, due to longer ectoparasitic feeding on root hairs than adults, $P$. penetrans juveniles penetrated the root later, and Zunke suggested that extended root hair feeding was essential for juveniles to obtain nutrients for further colonization. Because juveniles on agar can more easily migrate toward the root, perhaps less ectoparasitic feeding is required.

Root penetration zones. There are differences in the sites and mechanisms of root penetration by different species of Pratylenchus in various hosts. In lucerne and clover, $P$. penetrans preferred to penetrate the main root axes, where lateral root branches ruptured the cortex (63). These nematodes then migrated through the cortex of main root axes into other lateral root branches. Other studies suggested that most $P$. penetrans aggregated and penetrated in the zone of root elongation $(65,79)$. In lucerne, strawberry, and maize roots, the preferred zone of penetration was the dense root hairs (62). Zunke (80) observed $P$. penetrans penetrating and feeding on root hairs of various hosts, including rape, tobacco, and potato, but other studies indicated that $P$. penetrans only explored and penetrated the root hairs but did not feed on them $(32,41)$. However, Castillo et al. (11) suggested that $P$. thorne $i$ penetrated the roots of chickpea without any site preference. In the current study, $P$. thornei were commonly observed at the junction where lateral roots branched from the main seminal root. However, large groups were often also observed both in the zone of elongation and in seminal roots near the hypocotyl. Although penetration was not observed, $P$. thornei were seen within the dense root hair regions of both seminal and lateral roots. $P$. thornei may feed on root hairs and then move elsewhere along the root to penetrate. In this study, groups of $P$. thornei at various developmental stages, including eggs, aggregated in various root regions. These observations suggest that $P$. thornei does not have a preferred site of penetration in wheat.

Motility and migration. When toxic or unfavorable conditions arise, many organisms respond by becoming inactive by arresting development (45) or by becoming so damaged that they lose the 
ability to move. In this study, significantly higher numbers of $P$. thornei became nonmotile when exposed to REs (65\%) and CR suspensions (75\%) from resistant than susceptible (30\%) roots. Similarly, several other studies have reported suppressed motility in resistant roots and associated this response with accumulation of phytoalexins. For example, in response to invasion by $M$. incognita and Heterodera glycines, soybean synthesized the isoflavonoid glyceollin in the stele of resistant cultivars within 3 dai, which inhibited nematode respiration and motility $(23,28$, 29). Increased production of isoflavonoids in response to Pratylenchus invasion was also observed in several plant species. Coumestrol inhibited P. scribneri motility in resistant lima beans by $50 \%$ after exposure for 96 h (48). Similarly, high levels of medicarpin and coumestrol were found in resistant lucerne root tissue after invasion with $P$. penetrans, and motility was inhibited on agar by medicarpin (3). Despite the common link between isoflavonoids and Pratylenchus motility inhibition, in order to confirm the role of phytoalexins in providing resistance in the specific interaction between $P$. thornei and the resistance present in the Sokoll $\times$ Krichauff population, further work is required to identify specific compounds.

In both the RE and CR motility assays, some loss of nematode motility (20 to $30 \%$ ) occurred in the no-root water control, similar to that of the susceptible samples because, in general, motility is never $100 \%$ in nematode populations. The suppression of motility in the resistant lines was greater in the CR suspensions than in $\mathrm{RE}$, most likely due to the release of inhibitory compounds from vacuoles and other cellular compartments.
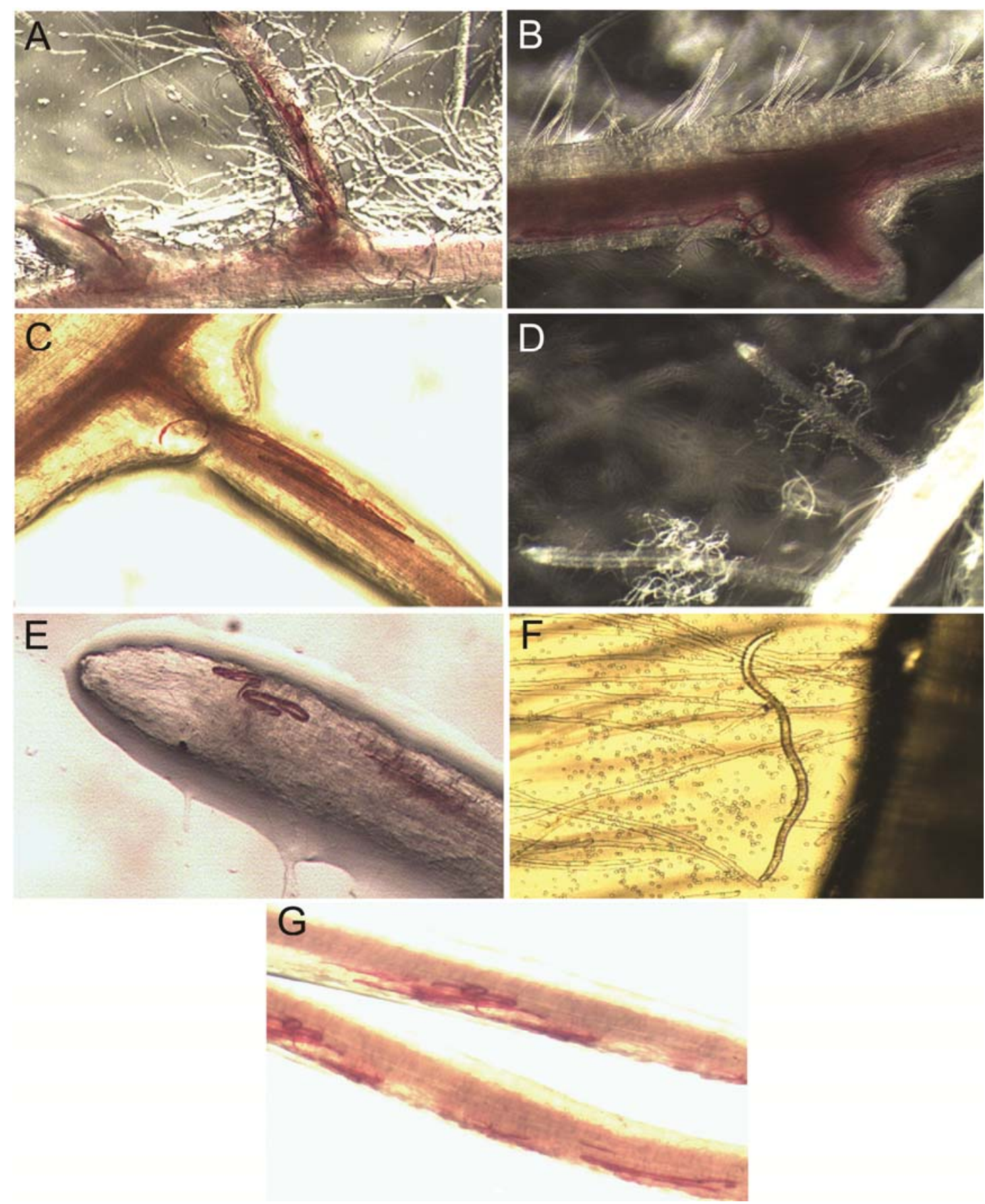

Fig. 5. Root penetration zones of Pratylenchus thornei in Triticum aestivum. A to C, Several P. thornei at lateral root branches. D, Groups of $P$. thornei probing and penetrating lateral roots. E, Several $P$. thorne $i$ at zone of elongation. F, Single $P$. thorne $i$ in the dense root hair zone. G, Aggregations of $P$. thornei juvenile and adult developmental stages within root regions. 
The effective RE and CR solutions were derived from seedlings not exposed to Pratylenchus spp. or other plant pathogens, which implies the presence of constitutively produced compounds in resistant roots that can suppress $P$. thornei motility. In the nematode Radopholus similis, temporary nonmotility was observed after exposure to RE from both susceptible and resistant banana cultivars that had not been infested with the nematode. In all, $\leq 50 \%$ were nonmotile after exposure to exudates from susceptible plants and the effects persisted for 1 to 3 days but $>80 \%$ of nematodes were nonmotile for $>6$ days in the case of exudates from resistant roots (76). The observed inhibitory effect on nematode motility of the resistant root compounds was partially reversible because $50 \%$ of the nonmotile $P$. thornei recovered motility when washed with water up to 2 days after exposure. A similar reversible effect within 2 days was described for P. scribneri exposed to coumestrol. However, after 4 days, few motile nematodes had recovered after washing (48). Based on the current results, the compounds involved in $P$. thornei motility suppression appear to be nematostatic but not nematicidal and affect $P$. thornei but not $P$. neglectus. Resistant genotypes caused inhibition of motility of 50 to $75 \%$; therefore, the role of these toxic compounds as defense mechanisms is significant. The motility assays designed in this study may provide useful alternative phenotypic resistance screening protocols.

Dual resistance to both $P$. thornei and $P$. neglectus is desirable because they commonly occur in wheat fields together (60). Generally, the investigated wheat sources of Pratylenchus resistance (AUS5205, AUS11984, and GS50a) do not contain resistance to both species at the same locus $(15,59)$. However, resistance QTL to both Pratylenchus spp. from the same wheat source (CPI133872) have been identified to co-locate (81), suggesting that, in some cases, resistance mechanisms to both species may share the same biological mechanism. The specificity of the suppressive root compounds identified in this study was assessed by performing motility assays with both $\mathrm{CR}$ suspensions and RE on $P$. neglectus. Results showed low motility suppression (25\%) in both resistant and susceptible roots. Although some loss in motility was observed, the proportion between the samples and the no-root water control was not significantly different. This

TABLE 3. Motility of Pratylenchus thornei 1 day after inoculation in different concentrations of root exudates collected from resistant (Sokoll) and susceptible (Krichauff) Triticum aestivum genotypes

\begin{tabular}{lccccc}
\hline & \multicolumn{2}{c}{ Sokoll } & & \multicolumn{2}{c}{ Krichauff } \\
\cline { 2 - 3 } \cline { 5 - 6 } $\begin{array}{l}\text { Root exudate } \\
(\mathrm{mg} / \mathrm{ml})\end{array}$ & $\begin{array}{c}\text { Nonmotile } \\
(\%)\end{array}$ & $\begin{array}{c}\text { Standard } \\
\text { error }\end{array}$ & & $\begin{array}{c}\text { Nonmotile } \\
(\%)\end{array}$ & $\begin{array}{c}\text { Standard } \\
\text { error }\end{array}$ \\
\hline 100 & 46.6 & 4.8 & & 32.6 & 7.9 \\
4 & 35.8 & 1.4 & & 16.3 & 4.7 \\
2 & 25.8 & 2.0 & & 22.4 & 2.6 \\
\hline
\end{tabular}

suggests that motility suppression caused by resistant root compounds investigated in this study is specific to P. thornei. Similar species specificity was observed with the inhibitory effect of coumestrol on $P$. scribneri but not on $M$. javanica, even at high concentrations $(25 \mu \mathrm{g} / \mathrm{ml})(48)$. Interestingly, the Sokoll $\times$ Krichauff population contains $P$. neglectus resistance, with Krichauff as the resistance source carrying the Rlnnl resistance gene (K. Linsell, unpublished data). However, as shown in this investigation, this resistance does not appear to be associated with motility suppression or is possibly not constitutively expressed.

Because it was established that resistant roots were able to suppress nematode motility in vitro, an experiment was designed to measure nematode migration in roots or, essentially, feeding within roots. The design meant that only a portion of one root was available for penetration, while the rest of the seedling's root

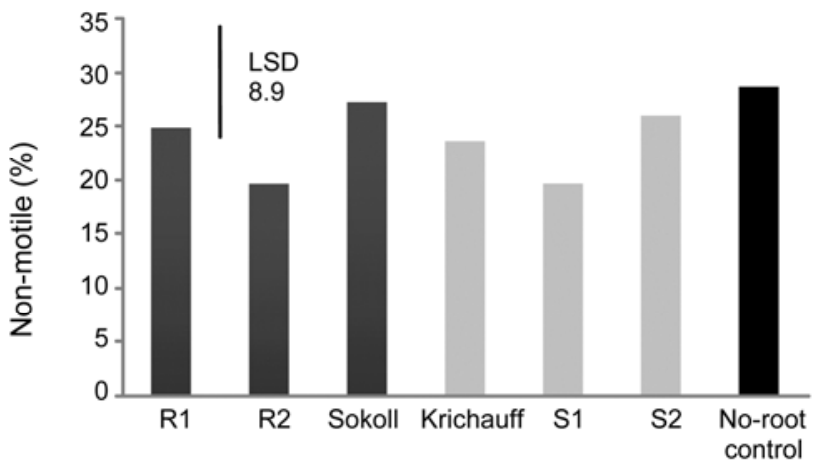

Fig. 7. Motility of Pratylenchus thornei in root exudates after 3 days of exposure from resistant (Sokoll, R1, and R2) and susceptible (Krichauff, S1, and S2) Triticum aestivum genotypes. LSD = least significant difference.

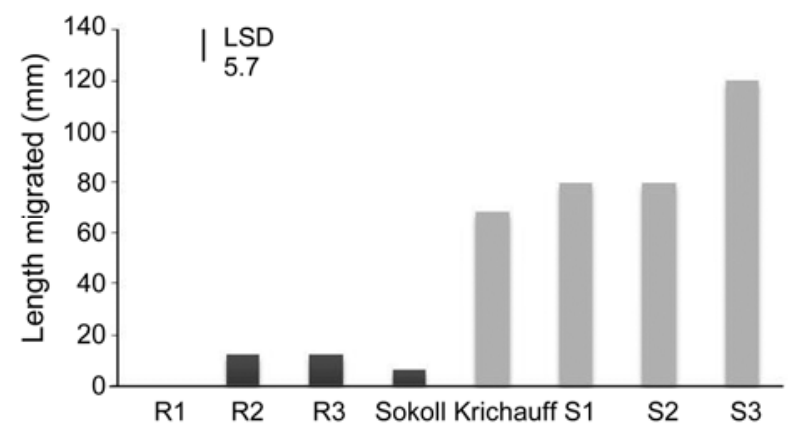

Fig. 8. Migration of Pratylenchus thornei within Triticum aestivum roots from the point of root inoculation, 10 days after inoculation in resistant (Sokoll, R1, R2, and R3) and susceptible (Krichauff, S1, S2, and S3) root genotypes. LSD $=$ least significant difference.
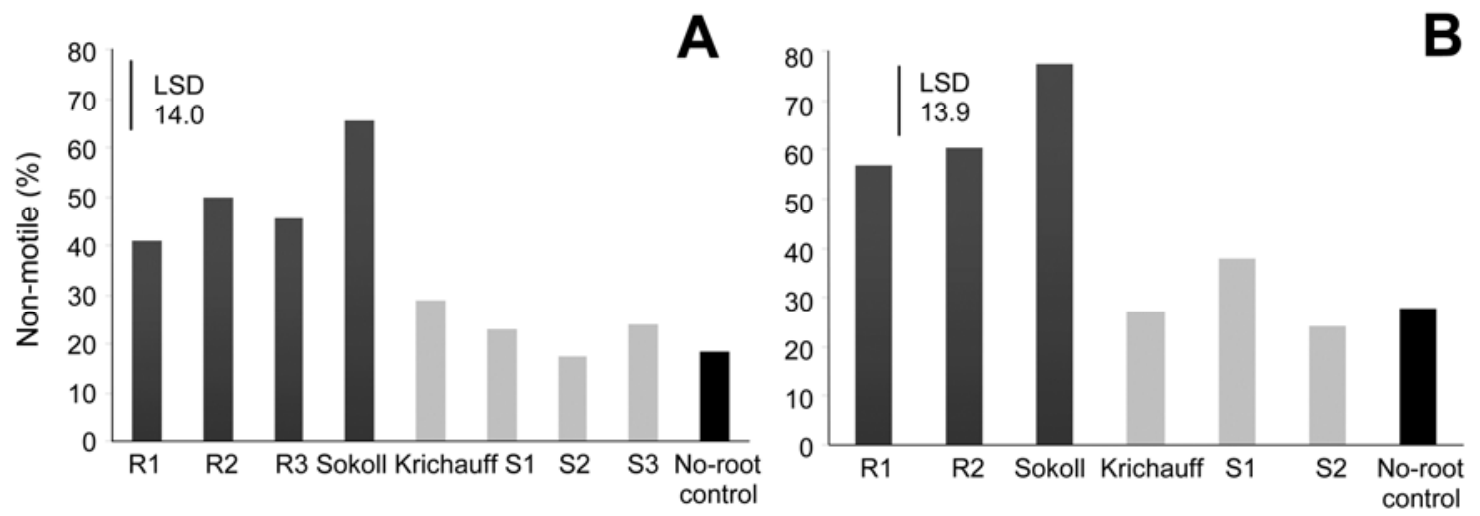

Fig. 6. Motility of Pratylenchus thornei in A, root exudates and B, crushed root suspensions from Triticum aestivum after exposure for 3 days. Genotypes assayed were resistant (Sokoll, R1, R2, and R3) and susceptible (Krichauff, S1, S2, and S3). LSD = least significant difference. 
system was physically blocked from the inoculated nematodes. These migration assays revealed that $P$. thornei did not migrate far through the root cortex from the point of inoculation in resistant roots. On average, nematodes were able to move $>60 \mathrm{~mm}$ further in susceptible Krichauff than the resistant Sokoll. Because $>50 \%$ penetration occurs within 9 hai on agar, root growth after inoculation will not affect the migration measured. It was essential that motility and migration assays were performed under sterile conditions because fungi are known to be sources of antinematodal compounds that can suppress motility and hatching $(66,72)$.

Reproduction and maturation. The developmental stages of $P$. thornei collected from inoculated roots at multiple times across
45 days matched well to the life-cycle observations made by Larson (33), the only other published report of $P$. thornei reproduction in wheat. Therefore, the development timeline reported in this study under glasshouse conditions can now be utilized in future studies of specific $P$. thornei reproductive stages. The analysis of development over time between the resistant and susceptible genotypes within the Sokoll $\times$ Krichauff population showed significantly less $P$. thornei at all stages in the resistant compared with the susceptible roots. Similarly, reduced reproduction was observed in a resistant durum wheat cultivar, where no difference in penetration between the susceptible and resistant cultivars also occurred (58). Reduced reproduction indicates that either the nematodes exit resistant roots or fail to develop and
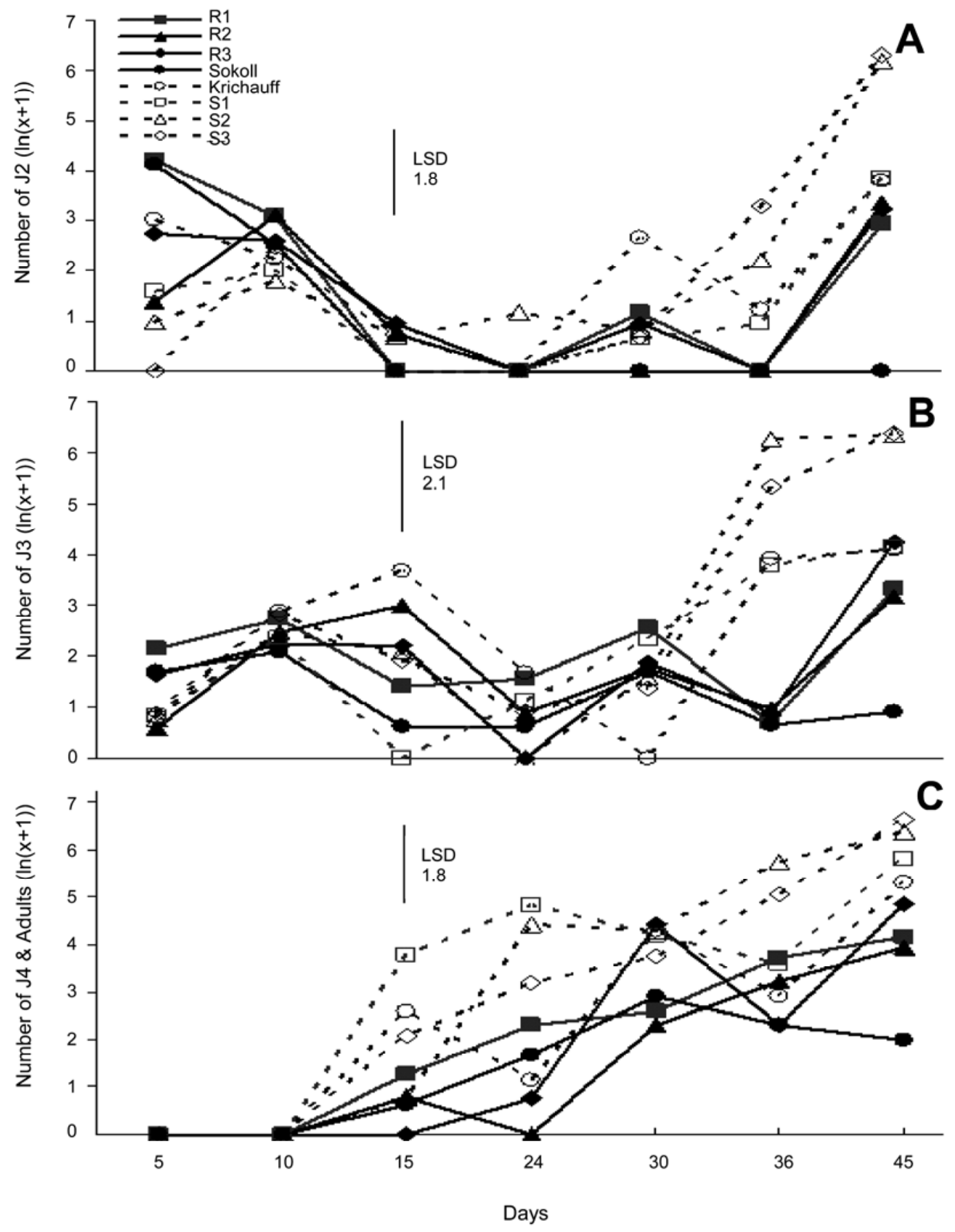

Fig. 9. Number (log transformed) of Pratylenchus thornei developmental stages within resistant (Sokoll, R1, R2, and R3) and susceptible (Krichauff, S1, S2, and S3) Triticum aestivum roots at different times within one nematode life cycle (45 days). A, Second-generation stage two juveniles (J2s) hatch at 36 days after inoculation (dai). B, Stage-three juveniles (J3s) first-generation molt at 10 dai and second generation at 45 dai. C, Stage-four juveniles (J4s) and adults firstgeneration molt at 14 to 28 dai. LSD = least significant difference. 
reproduce. This study showed that $P$. thornei nematodes do not exit roots because large numbers are still present in the roots even after 16 days, indicating that reproductive development is suppressed. In the second generation, up to 10 times as many J2s and $\mathrm{J} 3 \mathrm{~s}$ were detected in susceptible roots as in resistant roots, suggesting that molting is also suppressed or delayed in the resistant genotypes. This is supported by Farsi (14), who observed a delay in molting of $P$. neglectus in resistant wheat cultivars, with only $1.7 \%$ molting, compared with $30 \%$ in the susceptible. Delayed maturation was also observed in $P$. goodeyi in resistant banana roots. Adult and J4 development were delayed by 4 days in comparison with the susceptible cultivar, which affected optimum egg deposition and hatch (45). The current study shows that
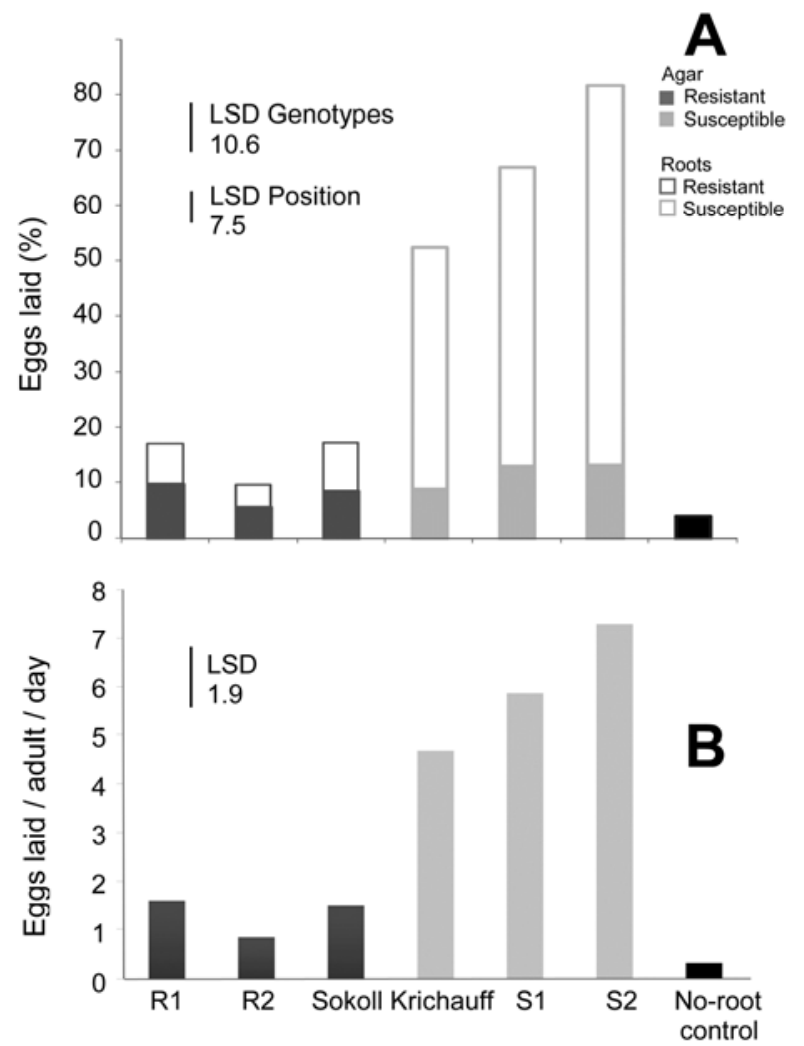

Fig. 10. Egg deposition of Pratylenchus thornei in and near Triticum aestivum seedling roots propagated on agar. A, Number of eggs deposited in (roots) and directly adjacent (agar) to resistant (Sokoll, R1, and R2) and susceptible (Krichauff, S1, and S2) root genotypes. B, Rate of eggs deposited per day in the resistant and susceptible genotypes. LSD = least significant difference. resistance expressed in the Sokoll $\times$ Krichauff population to $P$. thornei prevents juvenile maturation and, thus, reproduction.

Egg deposition and egg hatch. In addition to delayed maturation, experiments were conducted to determine whether egg deposition and hatching were also affected by the presence of resistant roots. Pratylenchus spp. deposit single eggs either in soil or within roots (40); both were examined in this study. Adult $P$. thornei did deposit eggs within and near resistant roots but egg deposition was up to 30 times less than in susceptible roots. Farsi (14) reported similar suppression for $P$. neglectus in resistant wheat cultivars, with egg deposition being delayed by 4 days compared with the susceptible cultivar and no eggs being observed in the investigated resistant cultivar even after 35 days.

There is limited data on the capacity of Pratylenchus spp. to deposit eggs (25). However, the maximum egg deposition rate of $P$. penetrans and P. brachyurus was 2.7 to 4.8 eggs/day (35), both over 11 days at $20^{\circ} \mathrm{C}$. In the current study, mean deposition rates for $P$. thornei were 4.7 eggs/day over 10 days at $21^{\circ} \mathrm{C}$ in Krichauff. This suggests that temperature, plant, and nematode species all affect the rate of egg deposition. The rate of egg deposition per female per day in the susceptible roots was almost three times higher than in the resistant roots. Thus, resistance appears to decrease the rate of egg deposition of $P$. thornei in the investigated wheat genotypes. In addition, fewer eggs were laid on agar plates where no roots were present, suggesting that Pratylenchus nematodes deposit eggs only when a food source is available. In susceptible plants, females preferentially deposited eggs within the roots $(83 \%)$ rather than outside. Only half the numbers of eggs were deposited within and adjacent to the resistant roots. A lower deposition of eggs within resistant roots

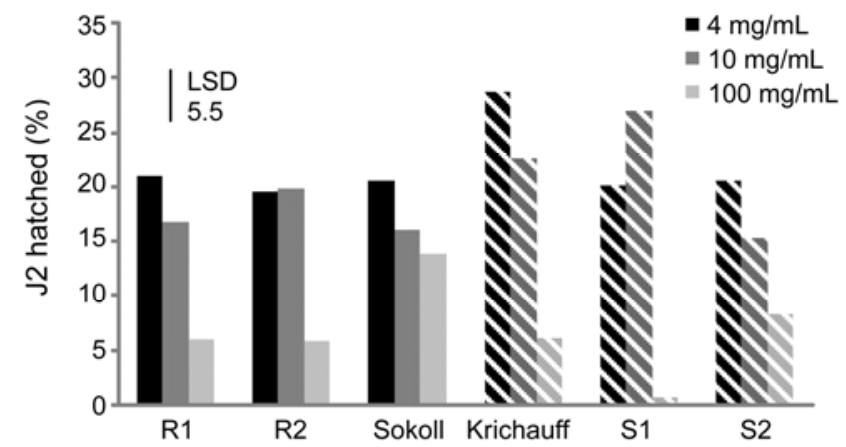

Fig. 12. Number of Pratylenchus thornei juveniles hatched from eggs soaked in serial dilutions of root exudates from resistant (Sokoll, R1, and R2) and susceptible (Krichauff, S1, and S2) Triticum aestivum genotypes. LSD = least significant difference.
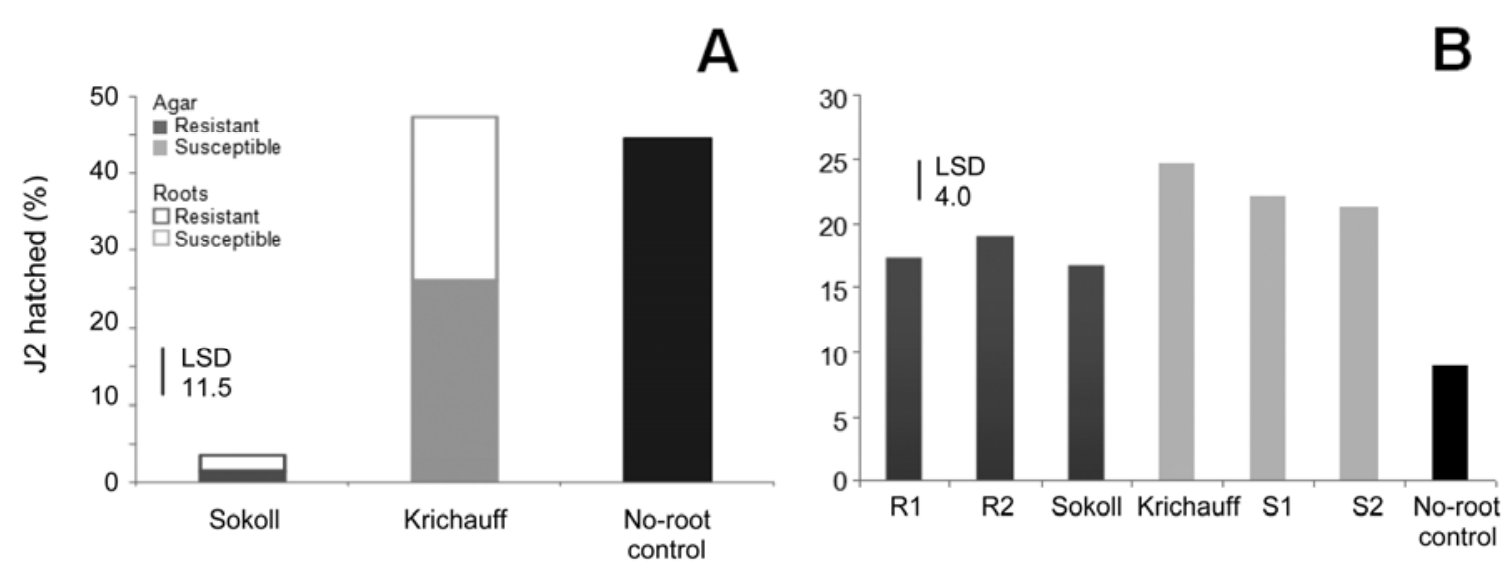

Fig. 11. Hatching of Pratylenchus thornei near Triticum aestivum seedling roots propagated A, on agar and $\mathbf{B}$, within root exudate solutions. A, Number of juveniles hatched within (roots) and directly near (agar) resistant (Sokoll) and susceptible (Krichauff) roots. B, Number of juveniles that hatched in resistant (Sokoll, R1, and R2) and susceptible (Krichauff, S1, and S2) root exudates after exposure for 3 days. LSD = least significant difference. 
may be due to a higher concentration of suppressive compounds or could be due to the reduced migration and feeding, so that mature females fail to acquire the energy to produce eggs. Reduced egg deposition has been associated with $P$. goodeyi resistance in banana where, in the susceptible roots, $P$. goodeyi nematodes deposited 29.8 eggs/day compared with 18.8 eggs/day in the resistant roots (45).

Hatching is a complex biological process affected by environmental factors and, sometimes, by the host $(54,55)$. In general, nematode hatching is significantly influenced by temperature, soil type, and moisture $(13,17,50,57)$. REs can contain hatching factors (HFs) that may stimulate hatching in some species of nematodes; and HIs, which prevent juvenile emergence from eggs. It is known that most species of nematodes do not solely rely on host-derived stimuli to initiate hatching and that many hatch freely in water (43), including $P$. thornei, as observed in this study. However, host signals are vital to ensure that significant numbers of juveniles emerge within a close vicinity of the roots to increase rates of successful penetration (43) or to induce hatch after a period of nematode dormancy (26). Byrne et al. (8) showed that hatch in response to potato root leachate of Globodera rostochiensis on agar correlated well with hatch in soil. Thus, hatching in this study was performed in vitro both on agar and in RE.

Significantly fewer $P$. thornei juveniles hatched from eggs within and adjacent to the roots of resistant versus susceptible seedlings grown on agar. Hatching was also decreased in resistant RE compared with susceptible but only by $8 \%$ after 10 days of exposure. The smaller hatching suppression observed in resistant RE compared with actively growing resistant roots on agar could be due to the presence of less $\mathrm{HI}$ in the $4 \mathrm{mg} / \mathrm{ml}$ concentration of $\mathrm{RE}$ used than that naturally exuded by live roots. In addition, it could be that the active compounds in RE are labile and become inactive over time (10 days). In the susceptible roots and in the no-root control, only $50 \%$ of $P$. thornei juveniles had emerged from deposited eggs after 10 days. Similarly, a maximum of $50 \%$ $P$. penetrans hatched even though $64 \%$ of the eggs already contained $\mathrm{J} 1 \mathrm{~s}$ or $\mathrm{J} 2 \mathrm{~s}$ during in vitro tests under optimum conditions (47). This suggests that despite uninterrupted juvenile maturation and deposition of eggs in favorable environmental conditions, not all juveniles will hatch. Similar suppression of hatching by resistant REs has been observed in other plant/ nematode interactions. RE from susceptible soybean cultivars stimulated more hatch and emergence of $H$. glycines than resistant cultivars $(9,54,55)$. Similarly, partially resistant clones of Solanum spp. hybrids generally stimulated lower hatch of $G$. pallida than susceptible cultivars after several weeks of exposure to RE (16). The flavonoids glyceollin and coumestrol, which suppressed $H$. glycines and $P$. scribneri motility as discussed earlier, also significantly affect egg hatch (64). Flavonoids or their derivatives such as ferulic, syringic, and coumeric acid are known to be present in wheat roots $(21,22,34,53)$ and could explain the hatching suppression in resistant REs observed in this investigation.

The observed resistance at hatching could be explained by a loss of an HF or the presence of HI. In addition to HF, there are HF stimulants, which are compounds that are hatch-neutral but can enhance the function of HF (8). The observation that a lower hatch rate occurred in resistant roots compared with the no-root control indicates the presence of HIs. The presence of HIs was further shown through dilution of the RE. Because HIs occur in low concentrations and HFs in much higher, dilution of RE should predominantly affect HIs without removing the activity of HFs (8). As the RE became more diluted, there was an increase in hatching in the resistant roots, thus further suggesting the presence of HIs for P. thornei. The observed decrease of hatching associated with the resistant wheat lines may be explained by inhibition or delay of egg hatch.
In conclusion, in the Sokoll $\times$ Krichauff population investigated, resistance acts post penetration with the suppression of migration, juvenile maturation, and, thus, reproduction. Consistent with various other plant-parasitic nematode species, there was no significant difference in the rate at which $P$. thornei was attracted toward or penetrated resistant or susceptible roots. No preferential root penetration zone was observed, unlike in other studies; however, once inside the root, groups of $P$. thornei at various developmental stages moved in the tracks left by previously migrating nematodes. Migration and motility of $P$. thornei is suppressed in resistant roots and exudates, suggesting that resistant genotypes constitutively produce compounds that inhibit motility. The effects of these compounds are reversible and differentially affect $P$. thornei but not $P$. neglectus.

Nematode multiplication was inhibited by resistance because juvenile maturation was suppressed in resistant roots, with very few developing past J3. P. thornei $\mathrm{J} 2 \mathrm{~s}$ were still present within the roots 10 dai; therefore, juvenile maturation is inhibited by resistance rather than causing the nematodes to exit resistant roots. In addition, egg deposition and hatch of $P$. thornei was significantly reduced in resistant roots and exudates. The increased hatch after dilution of RE and the lower hatch in resistant exudates versus the absence of roots indicates the presence of HIs. The actions of flavonoids and phenolics have been linked to Pratylenchus motility suppression in other resistance responses and, thus, are targets for future investigations into the resistance of $P$. thornei to Sokoll $\times$ Krichauff mapping population lines. Further characterization of biological resistance mechanisms and the development of robust screening protocols will enhance the development of $P$. thornei resistance breeding in wheat.

\section{ACKNOWLEDGMENTS}

This research was supported by the Grains Research and Development Corporation through a Grains Industry Research Scholarship to K. J. Linsell as part of the fulfillment of the requirements for the Degree of Doctor of Philosophy. The project was also supported by the South Australian Research and Development Institute (SARDI) and Molecular Plant Breeding Cooperative Research Center. We thank the SARDI Nematology Group, especially D. Pounsett, for assistance with culturing of the nematodes; and H. Wallwork at SARDI for access to plant material.

\section{LITERATURE CITED}

1. Abdel-Rahman, F. H., Alaniz, N. M., and Saleh, M. A. 2013. Nematicidal activity of terpenoids. J. Environ. Sci. Health 48:16-22.

2. Acedo, J. R., and Rhode, R. A. 1971. Histochemical root pathology of Brassica oleracea capitata L. infected by Pratylenchus penetrans (Cobb) Filipjev and Schuurmans Stekhoven (Nematoda: Tylenchidae). J. Nematol. 3:62-68.

3. Baldridge, G. D., O'Neill, N. R., and Samac, D. A. 1998. Alfalfa (Medicago sativa L.) resistance to the root-lesion nematode, Pratylenchus penetrans: Defense-response gene mRNA and isoflavonoid phytoalexin levels in roots. Plant Mol. Biol. 38:999-1010.

4. Balhadere, P., and Evans, A. A. F. 1994. Characterisation of attractiveness of excised root-tips of resistant and susceptible plants for Meloidogyne naasi. Fundam. Appl. Nematol. 17:527-536

5. Baxter, R. I., and Blake, C. D. 1967. Invasion of wheat roots by Pratylenchus thornei. Nature 215:1168-1169.

6. Berge, J. B. 1971. Méthodes culturales et variétés résistantes. Pages 537593 in: Les nématodes des cultures. Fédération Nationale des Groupements de Protection des Cultures (FNGPC), Paris.

7. Bybd, D. W., Kirkpatrick, T., and Barker, K. R. 1983. An improved technique for clearing and staining plant tissues for detection of nematodes. J. Nematol. 15:142-143.

8. Byrne, J., Twomey, U., Maher, N., Devine, K. J., and Jones, P. W. 1998. Detection of hatching inhibitors and hatching factor stimulants for golden potato cyst nematode, Globodera rostochiensis, in potato root leachate. Ann. Appl. Biol. 132:463-472.

9. Caballero, L. G., Osmanski, E. A., and Tefft, P. M. 1986. Factors influencing plant-induced egg hatching in Heterodera glycines. J. Nematol. 18:636.

10. Castillo, P., Trapero-Casas, J. L., and Jimenez-Diaz, R. M. 1995. Effect of 
time, temperature, and inoculum density on reproduction of Pratylenchus thornei in carrot disk cultures. J. Nematol. 27:120-124.

11. Castillo, P., Vovlas, N., and Jimenez-Diaz, R. M. 1998. Pathogenicity and histopathology of Pratylenchus thornei populations on selected chickpea genotypes. Plant Pathol. 47:370-376.

12. Creech, R. G., Jenkins, J. N., Tang, B., Lawrence, G. W., and McCarty, J. C. 1995. Cotton resistance to root-knot nematode .1. Penetration and reproduction. Crop Sci. 35:365-368.

13. Devine, K. J., and Jones, P. W. 2001. Effects of hatching factors on potato cyst nematode hatch and in-egg mortality in soil and in vitro. Nematology 3:65-74.

14. Farsi, M. 1996. Genetic variation for tolerance and resistance to Pratylenchus neglectus. Ph.D. thesis, Plant Science, University of Adelaide, Adelaide, Australia.

15. Farsi, M., Vanstone, V. A., Fisher, J. M., and Rathjen, A. J. 1995. Genetic variation in resistance to Pratylenchus neglectus in wheat and triticales. Aust. J. Exp. Agric. 35:597-602.

16. Forrest, J. M. S., and Phillips, M. S. 1984. The effect of Solanum tuberosum $\times$ Solanum vernei hybrids on hatching of the potato cyst nematode Globodera pallida. Ann. Appl. Biol. 104:521-526.

17. Gaur, H. S., and Haque, M. M. 1987. Effect of irrigation and stage of moisture stress on the growth of nematode populations under pea. Indian J. Nematol. 17:189-192.

18. Gheysen, G. 1998. Chemical signals in the plant-nematode interaction-a complex system? Pages 95-117 in: Phytochemical Signals and PlantMicrobe Interactions J. T. Romeo, K. R. Downum, and R. Verpoorte, eds. Plenum Press, New York

19. Griffin, G. D., and Elgin, J. H. 1977. Penetration and development of Meloidogyne hapla in resistant and susceptible alfalfa under differing temperatures. J. Nematol. 9:51-56.

20. Griffin, G. D., and Waite, W. W. 1971. Attraction of Ditylenchus dipsaci and Meloidogyne hapla by resistant and susceptible alfalfa seedlings. J. Nematol. 3:215.

21. Guenzi, W. D., and McCalla, T. M. 1966. Phenolic acids in oats, wheat, sorghum, and corn residues and their phytotoxicity. Agron. J. 58:303-304.

22. Guenzi, W. D., McCalla, T. M., and Norstadt, F. A. 1967. Presence and persistence of phytotoxic substances in wheat, oat, corn and sorghum residues. Agron. J. 59:163-165.

23. Huang, J. S., and Barker, K. R. 1986. Radioimmunoassay of glyceollin-I in soybean roots infected with race-1 of Heterodera glycines. Phytopathology 76:1139-1139.

24. Hussey, R. S. 1989. Disease-inducing secretions of plant-parasitic nematodes. Annu. Rev. Phytopathol. 27:123-141.

25. Inomoto, M. M., and Oliveira, C. M. G. 2008. Coffee associated Pratylenchus spp.-ecology and interactions with plants. Pages 51-64 in: Plant-Parasitic Nematodes of Coffee R. M. Souza, ed. Springer, Dordrecht, The Netherlands.

26. Jones, P. W., Tylka, G. L., and Perry, R. N. 1998. Hatching. Pages 181212 in: The Physiology and Biochemistry of Free-Living and PlantParasitic Nematodes R. N. Perry and D. J. Wright, eds. CAB International, Wallingford, UK.

27. Joshi, R. K. 2010. Gene pyramiding-a broad spectrum technique for developing durable stress resistance in crops. Biotechnol. Mol. Biol. Rev. 551 .

28. Kaplan, D. T., Keen, N. T., and Thomason, I. J. 1980. Association of glyceollin with the incompatible response of soybean roots to Meloidogyne incognita. Physiol. Plant Pathol. 16:309-318.

29. Kaplan, D. T., Keen, N. T., and Thomason, I. J. 1980. Studies on the mode of action of glyceollin in soybean-incompatibility to the root-knot nematode, Meloidogyne incognita. Physiol. Plant Pathol. 16:319-325.

30. Kim, Y. H., Riggs, R. D., and Kim, K. S. 1987. Structural changes with resistance of soybean to Heterodera glycines. J. Nematol. 19:177-187.

31. Kouassi, A. B., Kerlan, M. C., Sobczak, M., Dantec, J. P., Rouaux, C., Ellisseche, D., and Mugniery, D. 2004. Resistance to the root-knot nematode Meloidogyne fallax in Solanum sparsipilum: Analysis of the mechanisms. Nematology 6:389-400.

32. Kurrpa, S., and Vrain, T. C. 1985. Penetration and feeding behaviour of Pratylenchus penetrans in strawberry roots. Rev. Nématol. 8:273-276.

33. Larson, J. E. 1959. The biology of Pratylenchus thornei. Ph.D. thesis, University of California, Davis.

34. Lodhi, M. A. K., Bilal, R., and Malik, K. A. 1987. Allelopathy in agroecosystems: Wheat phytotoxicity and its possible roles in crop rotation. J. Chem. Ecol. 13:1881-1891.

35. Mamiya, Y. 1971. Effect of temperature on life cycle of Pratylenchus penetrans on Cryptomeria seedlings and observations on its reproduction. Nematologica 17:82-92.

36. McClure, M. A., Ellis, K. C., and Nigh, E. L. 1974. Post-infection development and histopathology of Meloidogyne incognita in resistant cotton. J. Nematol. 6:21-26.

37. Moens, M., and Perry, R. N. 2009. Migratory plant endoparasitic nematodes: A group rich in contrasts and divergence. Annu. Rev. Phytopathol. 47:313-332.

38. Moody, E. H., Lownsbery, B. F., and Ahmed, J. M. 1973. Culture of the root-lesion nematode Pratylenchus vulnus on carrot disks. J. Nematol. 19:125-134.

39. Murray, G. M., and Brennan, J. P. 2009. Estimating disease losses to the Australian wheat industry. Australas. Plant Pathol. 38:558-570.

40. Nicol, J. P., and Rivoal, R. 2008. Global knowledge and its application for the integrated control and management of nematodes on wheat. Pages 251-294 in: Integrated Management and Biocontrol of Vegetable and Grain Crops Nematodes. A. Ciancio and K. G. Mukerji, eds. Springer, Dordrecht, The Netherlands.

41. Ogiga, I. R., and Estey, R. H. 1975. Penetration and colonisation of Brassica rapa and Zea mays root tissues by Pratylenchus penetrans. Phytoprotection 56:22-30.

42. Ophel-Keller, K., McKay, A., Hartley, D., Herdina, and Curran, J. 2008. Development of a routine DNA-based testing service for soilborne diseases in Australia. Australas. Plant Pathol. 37:243-253.

43. Perry, R. N. 1989. Dormancy and hatching of nematode eggs. Parasitol. Today 5:377-383.

44. Perry, R. N. 1997. Plant signals in nematode hatching and attraction. Pages 38-50 in: Cellular and Molecular Aspects of Plant-Nematode Interaction. C. Fenoll, ed. Kluwer Academic Publishers, Boston.

45. Prasad, J. S., Seshu Reddy, K. V., and Sikora, R. A. 1999. Life cycle of Pratylenchus goodeyi in banana roots. Nematol. Mediterr. 27:111-114.

46. Prot, J. C. 1980. Migration of plant parasitic nematodes towards plant roots. Rev. Nématol. 3:305-318.

47. Pudasaini, M. P., Viaene, N., and Moens, M. 2008. Hatching of the rootlesion nematode, Pratylenchus penetrans, under the influence of temperature and host. Nematology 10:47-54.

48. Rich, J. R., Keen, N. T., and Thomason, I. J. 1977. Association of coumestans with the hypersensitivity of lima bean roots to Pratylenchus scriberni. Physiol. Plant Pathol. 10:105-116.

49. Robinson, M. P., Atkinson, H. J., and Perry, R. N. 1987. The influence of soil moisture and storage time on the motility, infectivity and lipid utilization of second stage juveniles of the potato cyst nematodes Globodera rostochiensis and G. Pallida. Rev. Nématol. 10:343-348.

50. Robinson, M. P., Atkinson, H. J., and Perry, R. N. 1987. The influence of temperature on the hatching, activity and lipid utilization of second stage juveniles of the potato cyst nematodes Globodera rostochiensis and G. pallida. Rev. Nématol. 10:349-354.

51. Rohde, R. A. 1972. Expression of resistance in plants to nematodes. Annu. Rev. Phytopathol. 10:233-252.

52. Roman, J., and Hirschmann, H. 1969. Morphology and morphometrics of six species of Pratylenchus. J. Nematol. 1 363-386.

53. Salomonsson, A. C., Theander, O., and Aman, P. 1978. Quantitative determination by GLC of phenolic acids as ethyl derivatives in cereal straw. J. Agric. Food Chem. 26:830-835.

54. Schmitt, D. P., and Riggs, R. D. 1991. Influence of selected plant species on hatching of eggs and development of juveniles of Heterodera glycines. J. Nematol. 23:1-6.

55. Sikora, E. J., and Noel, G. R. 1996. Hatch and emergence of Heterodera glycines in root leachate from resistant and susceptible soybean cultivars. J. Nematol. 28:501-509.

56. Southey, J. F. 1986. Laboratory Methods for Work with Plant and Soil Nematodes. Her Majesty's Stationery Office, London.

57. Stoyanov, D., and Trifonova, Z. 1995. Hatching dynamics of golden potato cyst nematode larvae Globodera rostochiensis Woll. Bulg. J. Agric. Sci. 1:241-246.

58. Talavera, M., and Vanstone, V. A. 2001. Monitoring Pratylenchus thornei densities in soil and roots under resistant (Triticum turgidum durum) and susceptible (Triticum aestivum) wheat cultivars. Phytoparasitica 29:29-35.

59. Thompson, J. P., Brennan, P. S., Clewett, T. G., Sheedy, J. G., and Seymour, N. P. 1999. Progress in breeding wheat for tolerance and resistance to root-lesion nematode (Pratylenchus thornei). Australas. Plant Pathol. 28:45-52.

60. Thompson, J. P., Clewett, T. G., Sheedy, J. G., Reen, R. A., O’Reilly, M. M., and Bell, K. L. 2010. Occurrence of root-lesion nematodes (Pratylenchus thornei and P. neglectus) and stunt nematode (Merlinius brevidens) in the northern grain region of Australia. Australas. Plant Pathol. 39:254-264.

61. Thompson, J. P., Owen, K. J., Stirling, G. R., and Bell, M. J. 2008. Rootlesion nematodes (Pratylenchus thornei and P. neglectus): a review of recent progress in managing a significant pest of grain crops in northern Australia. Australas. Plant Pathol. 37:235-242.

62. Townshend, J. L. 1978. Infectivity of Pratylenchus penetrans on alfalfa. J. Nematol. 10:318-323.

63. Townshend, J. L., Stobbs, L., and Carter, R. 1989. Ultrastructural pathology of cells affected by Pratylenchus penetrans in alfalfa roots. J. Nematol. 21:530-539. 
64. Trivedi, P. C., Barker, K. R., and Huang, J. S. 1984. Effects of glyceollin on hatching and motility of Heterodera glycines. Phytopathology 74:830830.

65. Troll, J., and Rhode, R. A. 1966. Pathogenicity of Pratylenchus penetrans and Tylenchorhynchus claytoni on turfgrasses. Phytopathology 56:995998.

66. Twomey, U., Warrior, P., Kerry, B. R., and Perry, R. N. 2000. Effects of the biological nematicide, DiTera, on hatching of Globodera rostochiensis and G. pallida. Nematology 2:355-362.

67. Vanstone, V. A., Hollaway, G. J., and Stirling, G. R. 2008. Managing nematode pests in the southern and western regions of the Australian cereal industry: continuing progress in a challenging environment. Australas. Plant Pathol. 37:220-234. doi:10.1071/ap08020

68. Viglierchio, D. R. 1961. Attraction of parasitic nematodes by plant root emanations. Phytopathology 51:136-142.

69. Wallace, H. R. 1968. Dynamics of nematode movement. Annu. Rev. Phytopathol. 6:91.

70. Wallace, H. R. 1974. Nematology Ecology and Plant Disease. E. Arnold, ed. E. Arnold, London.

71. Wallace, H. R. 1989. Environment and plant health: A nematological perception. Annu. Rev. Phytopathol. 27:59-75.

72. Warrior, P., Rehberger, L. A., Beach, M., Grau, P. A., Kirfman, G. W., and Conley, J. M. 1999. Commercial development and introduction of DiTera, a new nematicide. Pestic. Sci. 55:376-379.

73. Weiser, G. C., Mueller, J. D., and Shipe, E. R. 1987. Response of tolerant and susceptible soybean cultivars to Columbia lance nematode. Pages 260-262 in: Soybean Genetics Newsletter. U.S. Dep. Agric. Agric. Res.
Serv. 14:260-262.

74. Williams, K. J., and Fisher, J. M. 1993. Development of Heterodera avenae Woll and host cellular-responses in susceptible and resistant wheat. Fundam. Appl. Nematol. 16:417-423.

75. Williamson, V. M., and Hussey, R. S. 1996. Nematode pathogenesis and resistance in plants. Plant Cell 8:1735-1745.

76. Wuyts, N., Swennen, R., and De Waele, D. 2006. Effects of plant phenylpropanoid pathway products and selected terpenoids and alkaloids on the behaviour of the plant-parasitic nematodes Radopholus similis, Pratylenchus penetrans and Meloidogyne incognita. Nematology 8:89101.

77. Zhao, X., Schmitt, M., and Hawes, M. 2000. Species-dependent effects of border cell and root tip exudates on nematode behaviour. Nematology 90:1239-1245.

78. Zuckerman, B. M., and Jansson, H. B. 1984. Nematode chemotaxis and possible mechanisms of host prey recognition. Annu. Rev. Phytopathol. 22:95-113.

79. Zunke, U. 1990. Observations on the invasion and endoparasitic behavior of the root lesion nematode Pratylenchus penetrans. J. Nematol. 22:309320 .

80. Zunke, U. 1990. Ectoparasitic feeding behaviour of the root lesion nematode, Pratylenchus penetrans, on root hairs of different host plants. Rev. Nématol. 13:331-337.

81. Zwart, R. S., Thompson, J. P., and Godwin, I. D. 2005. Identification of quantitative trait loci for resistance to two species of root-lesion nematode (Pratylenchus thornei and P. neglectus) in wheat. Aust. J. Agric. Res. 56:345-352. 